\section{Establishment of strigolactone-producing bacterium-yeast consortium}

\author{
Sheng $\mathrm{Wu}^{1 \dagger}$, Xiaoqiang $\mathrm{Ma}^{2 \dagger}$, Anqi Zhou ${ }^{1}$, Alex Valenzuela ${ }^{3}$, Kang Zhou ${ }^{2,4 *}$, Yanran $\mathrm{Li}^{1 *}$
}

Strigolactones (SLs) are a class of phytohormones playing diverse roles in plant growth and development, yet the limited access to SLs is largely impeding SL-based foundational investigations and applications. Here, we developed Escherichia coli-Saccharomyces cerevisiae consortia to establish a microbial biosynthetic platform for the synthesis of various SLs, including carlactone, carlactonoic acid, 5-deoxystrigol (5DS; $6.65 \pm 1.71 \mu \mathrm{g} /$ liter), 4-deoxyorobanchol ( $3.46 \pm 0.28 \mu \mathrm{g} /$ liter), and orobanchol (OB; $19.36 \pm 5.20 \mu \mathrm{g} / \mathrm{liter})$. The SL-producing platform enabled us to conduct functional identification of CYP722Cs from various plants as either OB or 5DS synthase. It also allowed us to quantitatively compare known variants of plant SL biosynthetic enzymes in the microbial system. The titer of 5DS was further enhanced through pathway engineering to $47.3 \mu \mathrm{g} / \mathrm{liter}$. This work provides a unique platform for investigating SL biosynthesis and evolution and lays the foundation for developing SL microbial production process.

\section{INTRODUCTION}

Strigolactones (SLs) were initially characterized as signaling molecules, which are released from plant roots, induce germination of root parasitic weed, regulate the hyphae branching of arbuscular mycorrhiza fungi (AMF), and promote the symbiotic relationship between plants and fungi $(1,2)$. Later, they were also identified as a previously unknown class of plant hormones that control shoot branching, leaf growth, and senescence and promote the formation of lateral root and growth of primary root (3). SLs thus have been considered as promising agrochemicals, such as biostimulants that enhance the nutrient uptake efficiency through modulating plantAMF symbiotic association (4-6). To date, more than 30 natural SLs have been isolated (fig. S1) $(7,8)$. SLs generally consist of a conserved butenolide ring ( $\mathrm{D}$ ring) connected to a less conserved tricyclic lactone ring via an enol-ether bond (Fig. 1) (9). They can be classified into canonical and noncanonical SLs: The canonical SLs contained the tricyclic lactone-ring (ABC ring), while the noncanonical SLs lack the tricyclic ring scaffold with one (C ring) or two rings (B ring and $\mathrm{C}$ ring) missing (10). The canonical SLs can be further subdivided into orobanchol $(O)$ - and strigol (S)-type SLs according to the stereochemistry in the $\mathrm{C}$ ring, which are represented by 4-deoxyorobanchol (4DO) and 5-deoxystrigol (5DS), respectively (9). Some of the better-known noncanonical SLs include methylcarlactonoate (MeCLA) (11), heliolactone (12), avenaol (13), zealactone (14), and lotuslactone (15).

SLs are derived from all-trans- $\beta$-carotene (AT $\beta C)$, which is converted to carlactone (CL), the key branching point in SL biosynthesis (16), by the functions of three plastid enzymes: the isomerase DWARF27 (D27) and carotenoid cleavage dioxygenases 7 and 8 (CCD7 and CCD8) (Fig. 1) (16). D27, a [2Fe-2S]-containing polypeptide, catalyzes the isomerization of AT $\beta \mathrm{C}$ to 9 -cis- $\beta$-carotene (9C $\beta C)(16,17)$, followed by CCD7, a non-heme iron-dependent

\footnotetext{
'Department of Chemical and Environmental Engineering, University of California, Riverside, Riverside, CA 92521, USA. ${ }^{2}$ Disruptive and Sustainable Technologies for Agricultural Precision, Singapore-MIT Alliance for Research and Technology, Singapore, Singapore. ${ }^{3}$ Department of Botany and Plant Sciences, University of California, Riverside, Riverside, CA 92521, USA. ${ }^{4}$ Department of Chemical and Biomolecular Engineering, National University of Singapore, Singapore, Singapore.

*Corresponding author. Email: yanranl@ucr.edu (Y.L.); kang.zhou@nus.edu.sg (K.Z.) tThese authors contributed equally to this work.
}

enzyme that catalyzes the $\mathrm{C}^{\prime}-\mathrm{C} 10^{\prime}$ double bond cleavage of $9 \mathrm{C} \beta \mathrm{C}$ to yield 9 -cis- $\beta$-apo- $10^{\prime}$-carotenal $\left(9 \mathrm{C} \beta A 10^{\prime} \mathrm{CAL}\right)$ and $\beta$-ionone $(\beta \mathrm{I})$ $(16,18,19)$. Subsequently, CCD8 further catalyzes the oxidative cleavage of $9 \mathrm{C} \beta \mathrm{A} 10^{\prime} \mathrm{CAL}$ to synthesize $\mathrm{CL}$, with the reaction mechanism remaining elusive $(16,20)$. CL is then exported into cytoplasm and further oxidized by cytochrome P450s (CYPs) and other oxidases to afford various SL structures (21). The first oxidation step has been characterized to be the C19 oxidation of CL to synthesize carlactonoic acid (CLA), which can be catalyzed by the MORE AXILLARY GROWTH 1 (MAX1), a member of the CYP711A subfamily (11).

MAX1 is conserved in a number of plant species (22) and has many homologs. Rice, known to produce $O$-type SL such as $4 \mathrm{DO}$ and orobanchol (OB) (23), encodes five MAX1 homologs. One MAX1 homolog, OsCYP711A2 encoded by Os900, was identified to catalyze the conversion of CL to $4 \mathrm{DO}$ likely via CLA $(22,24)$. The $\mathrm{B}$ ring of $4 \mathrm{DO}$ can be further oxidized by MAX1 analogs (OsCYP711A3 encoded by $O s 1400$ from rice and $\mathrm{ZmMAX} 1 \mathrm{~b}$ from maize) to afford OB (22). However, 4DO is not produced in many OB-producing plants (e.g., cowpea), which hints another synthetic route of $O B$ without involving $4 \mathrm{DO}(25,26)$. This direct synthesis of $\mathrm{OB}$ from CLA was later identified to be catalyzed by CYP722C in cowpea (27). Recently, a CYP722C from cotton was characterized to catalyze the synthesis of 5DS from CLA (28).

Biosynthesis of most other SLs have not been fully elucidated. Studying these pathways could be sped up if total biosynthesis of key SLs and pathway intermediates (e.g., CL, CLA, OB, 4DO, and 5DS) from sugar can be established in fast-growing microbial hosts. In situ-produced SLs and pathway intermediates in the microbial system can serve as substrates to characterize recombinant SL biosynthetic enzyme candidates, eliminating the need of acquiring these molecules from plant materials or chemical synthesis. On the other hand, much remains to be investigated to understand the evolutionary history of SL biosynthesis and signaling in plants, especially nonvascular plants. The SL biosynthetic genes are either missing from the nonvascular plants or quite distinct from the corresponding variants encoded by the vascular plants (29). For example, although Charales (green algae) have been reported to produce SLs (e.g., sorgolactone) (30), no close CCD7 and CCD8 analogs have been found from either expression profiles or genomes, whether Charales can synthesize SL or not remains in debate (29). 


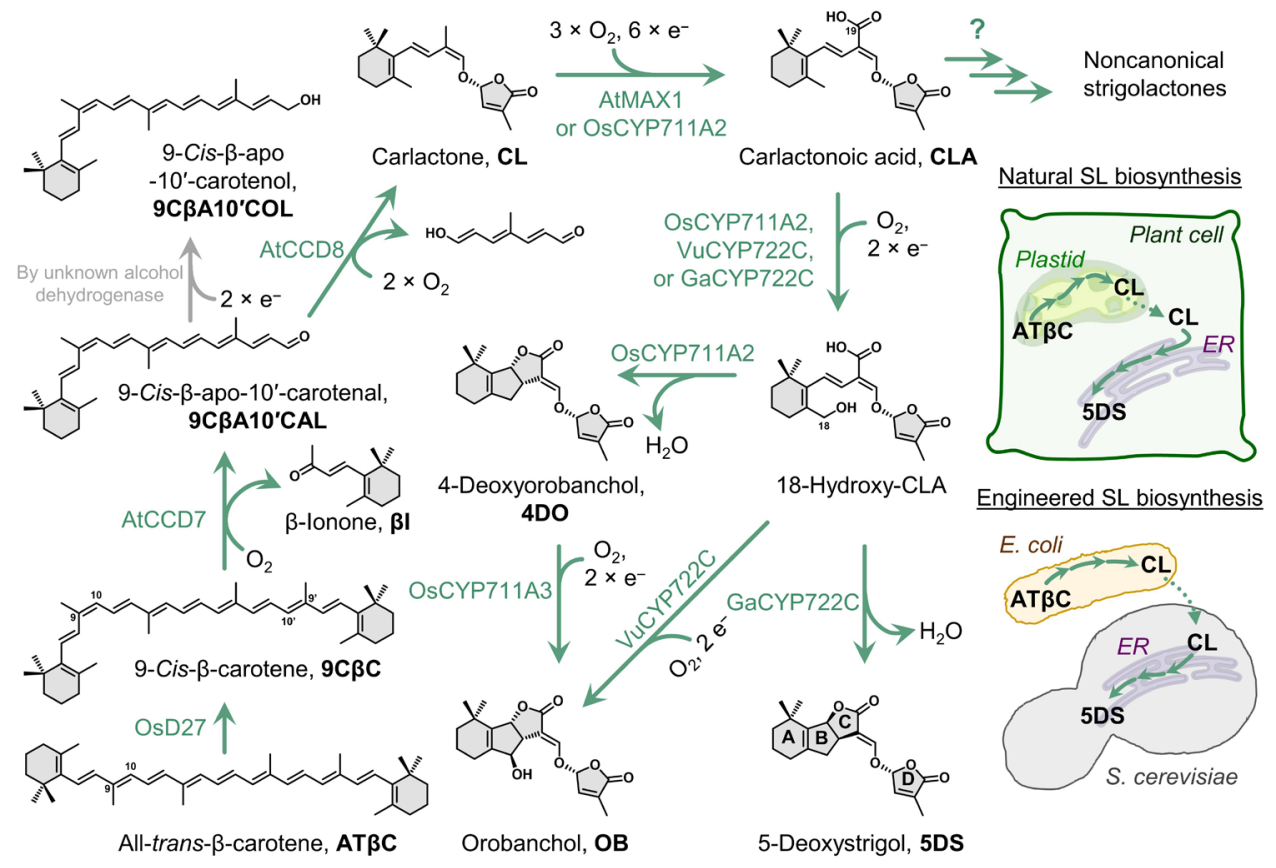

Fig. 1. Mimicking plant strigolactone pathway distribution in the engineered E. coli-S. cerevisiae coculture. OsD27, D27 from 0. sativa; AtCCD7, CCD7 from A. thaliana; AtCCD8, CCD8 from A. thaliana; AtMAX1, MORE AXILLARY GROWTH 1 from A. thaliana; OsCYP711 A2, cytochrome P450 CYP711A2 from O. sativa; OsCYP711 A3, cytochrome P450 CYP711A3 from O. sativa; VuCYP722C, cytochrome P450 CYP722C from V. unguiculata; GaCYP722C, cytochrome P450 CYP722C from G. arboreum. Carlactone is an important branch point. NADPH-cytochrome P450 reductase 1 from A. thaliana (ATR1) is expressed in yeast for the functional reconstitution of the plant cytochrome P450s. ER, endoplasmic reticulum.

An efficient functional identification strategy aided by a microbial SL-producing platform can also advance understanding of SL origin and evolution.

In this study, we first attempted to establish the SL biosynthetic pathway in Saccharomyces cerevisiae but failed, possibly due to the unsuccessful functional reconstitution of D27 and CCD7. We then tested the use of Escherichia coli as the host, since the active form of D27, CCD7, and CCD8 have been heterologously expressed and isolated from $E$. coli before $(16,18-20,31)$. An engineered $E$. coli strain successfully produced CL but failed to further functionalize it. The challenge was later overcome by using mixed cultures of $E$. coli and $S$. cerevisiae. The total biosynthesis of OB, 4DO, and 5DS was achieved from xylose. We used this microbial biosynthetic platform to identify eight CYP722Cs that produced either OB or 5DS from CLA and established a sequence-function correlation that could be used to predict the function of unknown CYP722Cs. Next, we chose $5 \mathrm{DS}$ as a model SL and improved the titer in the microbial consortium by $220 \%$ to $47.3 \mu \mathrm{g} /$ liter (shake flask culture) through metabolic engineering. The improved titer could be useful to isolate adequate pathway intermediates in future studies. Last, we quantitatively compared variants of D27, CCD7, and CCD8 from different plant species in the optimized system.

\section{RESULTS}

\section{It is challenging to establish CL production in S. cerevisiae}

To synthesize CL, we first attempted to functionally reconstitute D27 and CCD7 in a AT $\beta C$-producing $S$. cerevisiae strain. We reconstructed the AT $\beta C$-producing yeast strain (YYL23; table S2) as previously described (32). Trace amount of $9 \mathrm{C} \beta \mathrm{C}$ naturally existed in the established AT $\beta C$-producing yeast strain with a ratio to the AT $\beta C$ approximately 1:25 (fig. S2A). Expressing D27 from Arabidopsis thaliana (AtD27) or rice (OsD27) did not increase the ratio (fig. S2A). We extensively explored commonly used strategies for functionally reconstituting D27 (protein N-terminal engineering, protein localization to mitochondria, improving [2Fe-2S] cluster biogenesis, and reducing oxidative stress; fig. S2, A to C), but without success. We also tried to reconstitute the function of CCD7 from $A$. thaliana due to the presence of the substrate of CCD7, 9CßC, although at a low titer in YYL23. Although CCD1 has been functionally expressed in yeast to cleave $\beta$-carotene to afford the synthesis of $\beta I$ (33), we did not detect any activity of CCD7 possibly due to the fact that the CCD1 is natively localized in cytosol, while the other CCDs are in plastid (fig. S2D) (34). Truncating $\mathrm{N}$ terminus of CCD7 and/or targeting it to yeast mitochondria, which resemble plastids to certain extent, did not lead to detection of CCD7 activity in the yeast (fig. S2D).

\section{Establishment of CL production in $E$. coli}

Previous investigations indicate that D27, CCD7, and CCD8 can be expressed and isolated in soluble form from $E$. coli for the in vitro biochemical investigations $(16,18-20,31)$. Thus, we shifted the CL production from yeast to E. coli. First, OsD27 was expressed from a medium copy number plasmid $p C D F D u e t-1$ (table S1) in E. coli, under the control of $T 7$ promoter, in the presence of the well-documented AT $\beta C$-producing plasmid $p A C$-BETAipi (35) (resulting strain ECL-2, $E$. coli harboring $p A C-B E T A i p i$ to produce $A T \beta C$ is designated as ECL-1; table S2). Upon the introduction of OsD27, the ratio between $9 \mathrm{C} \beta \mathrm{C}$ to $\mathrm{AT} \beta \mathrm{C}$ was increased from 1:4.1 to 1:1.3, which indicates the functional reconstitution of OsD27 in E. coli (Fig. 2A and fig. S3, A and 

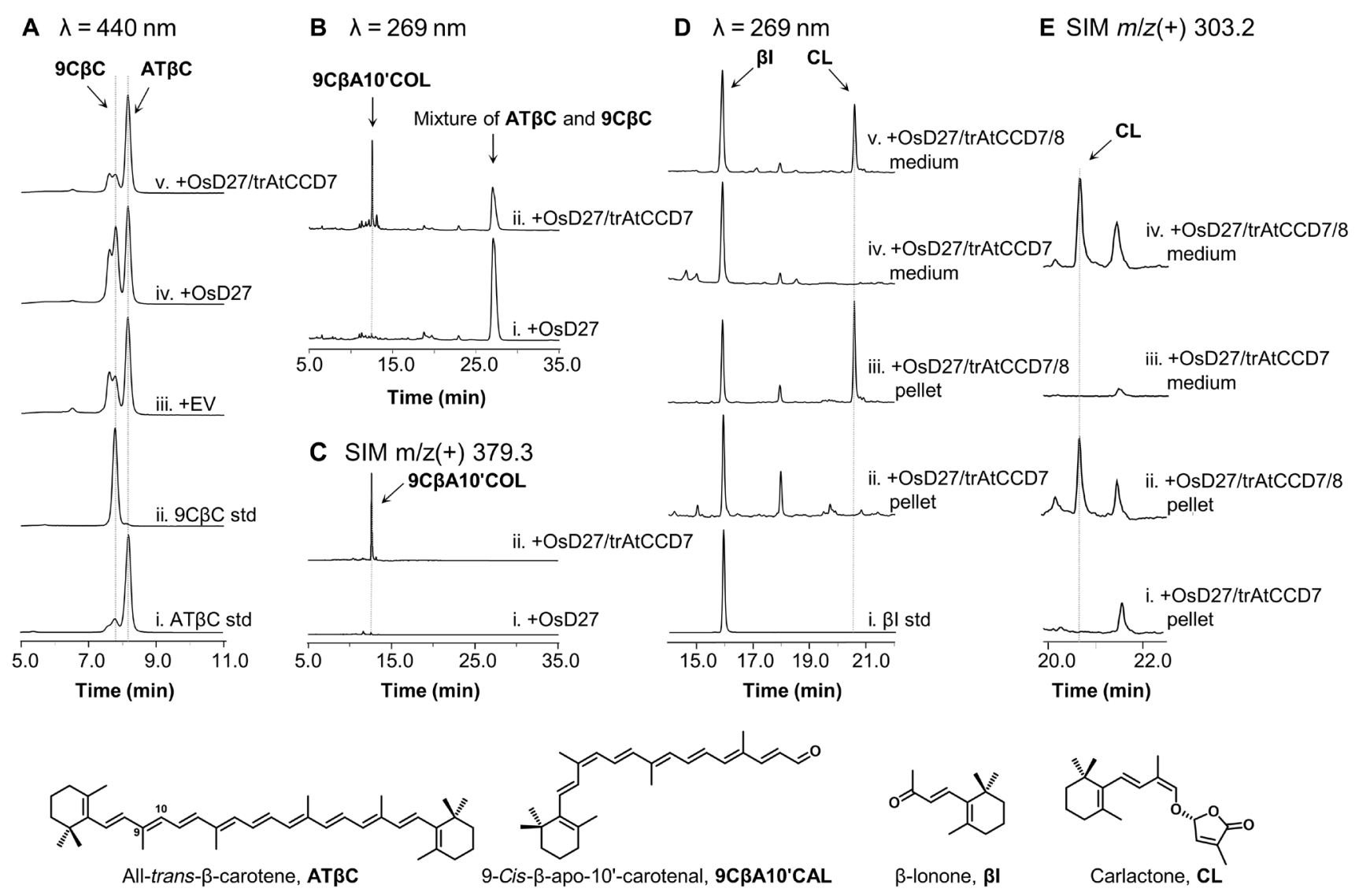

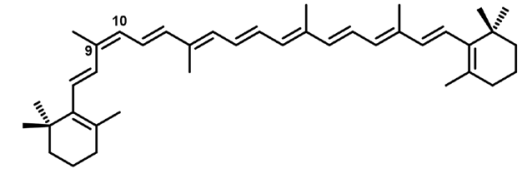

9-Cis- $\beta$-carotene, $9 \mathrm{C} \beta \mathrm{C}$<smiles>CC(C)=CC=CC(C)=CC=CC(C)=CC=CCO</smiles>

9-Cis- $\beta$-apo-10'-carotenol, 9CßA10'COL

Fig. 2. Production of $\mathrm{CL}$ in $E$. coli. Information of all the microbial strains mentioned in the caption can be found in table $S 2$. (A) High-performance liquid chromatography (HPLC) analysis $(\lambda=440 \mathrm{~nm}$ ) of (i) ATBC standard, (ii) $9 C \beta C$ standard, cell extracts of $E$. coli harboring (iii) AT $\beta C$-producing plasmid ( $p A C$-BETAipi; table S1) and empty plasmid (EV) (ECL-1), (iv) pAC-BETAipi and OsD27-expressing plasmid (ECL-2), and (v) pAC-BETAipi and OsD27/trAtCCD7 coexpressing plasmid (ECL-3). The samples were analyzed using the Separation Method I (see Materials and Methods). (B) HPLC analysis ( $\lambda=390 \mathrm{~nm}$ ) of cell extracts of (i) ECL-2 and (ii) ECL-3 by using the Separation Method II (see Materials and Methods). (C) Selected ion monitoring (SIM) extracted ion chromatogram (EIC) at 9 C $\beta A 10^{\prime} \mathrm{COL}$ 's characteristic $\mathrm{m} / \mathrm{z}^{+}=379.3$ of cell extracts of (i) ECL-2 and (ii) ECL-3. Separation Method II was used. (D) HPLC analysis ( $\lambda=269 \mathrm{~nm}$ ) of (i) $\beta 1$ standard, 9 C $\beta A 10^{\prime} C O L$-producing E. coli harboring (ii) EV (cell pellet extracts, ECL-4 N), (iii) trAtCCD8-expressing plasmid (cell pellet extracts, ECL-4), (iv) ECL-4N (medium extracts), and (v) ECL-4 (medium extracts) using the Separation Method III (see Materials and Methods). (E) SIM EIC at CL's characteristic m/z signal [molecular weight (MW) $=302.41$, $\left.\left[\mathrm{C}_{19} \mathrm{H}_{26} \mathrm{O}_{3}+\mathrm{H}\right]^{+}=\left[\mathrm{C}_{19} \mathrm{H}_{27} \mathrm{O}_{3}\right]^{+}=303.2\right]$ of (i) ECL-4 N (cell pellet extracts), (ii) ECL-4 (cell pellet extracts), (iii) ECL-4 N (medium extracts), and (iv) ECL-4 (medium extracts). Separation Method III was used. All traces are representative of at least three biological replicates for each engineered $E$. coli strain.

B). In the presence of OsD27, the titer of $9 \mathrm{C} \beta \mathrm{C}$ was $1.41 \mathrm{mg} / \mathrm{liter}$. The activity of D27 from A. thaliana (AtD27) was also examined in ECL-1 (ECL-2"; table S2), which exhibited a lower activity than OsD27 (fig. S4). We truncated the putative plastid transit peptide (first 40 amino acids) from OsD27 but did not detect obvious enhancement in the conversion toward 9С $\beta \mathrm{C}$ (fig. S4). Previous investigation on D27 indicated that this $\beta$-carotene isomerase catalyzes reversible conversion between $\mathrm{AT} \beta \mathrm{C}$ and $9 \mathrm{C} \beta \mathrm{C}$ (31), and thus, the ratio between 9 C $\beta C$ to AT $\beta C$ in ECL-2 might have reached the equilibrium.

Subsequently, the $\mathrm{N}$ terminus of CCD7 from A. thaliana (AtCCD7) was truncated [first 31 residues (31), trAtCCD7] and introduced to $9 \mathrm{C} \beta \mathrm{C}$-producing ECL-2 from the same plasmid
pCDFDuet-1-expressing OsD27, under the control of $T 7$ promoter (ECL-3; table S2). Liquid chromatography-mass spectrometry (LC-MS) analysis indicated the synthesis of a compound with a mass/charge ratio $(\mathrm{m} / \mathrm{z})$ positive $=379.3$ that agrees with 9 -cis $-\beta$ apo-10' -carotenol (9CBA10'COL) (Fig. 2, B and C, and fig. S3C) $(16,18,19)$. Although the product of CCD7 in vitro is $9 \mathrm{C} \beta A 10^{\prime} \mathrm{CAL}$ (16), the aldehyde can be reduced to the corresponding alcohol, $9 \mathrm{C} \beta \mathrm{A} 10^{\prime} \mathrm{COL}$, in $E$. coli $(18,19)$. The synthesis of $\beta \mathrm{I}$, the other product of CCD7, was confirmed through comparison with the authentic standard (figs. S3D and S5). No 9C $\beta A 10^{\prime} \mathrm{COL}$ (Fig. 2C) but a trace amount of $\beta I$ was detected in ECL-2 (fig. S5), and $\beta \mathrm{I}$ is believed to be produced by the autoxidation of AT $\beta C$ (36). 
The N-terminal truncated (first 56 residues) AtCCD8 (trAtCCD8) was then expressed in the $9 \mathrm{C} \beta \mathrm{A} 10^{\prime} \mathrm{COL}$-producing ECL-3 from a medium copy number plasmid pET21a under the control of $T 7$ promoter (ECL-4; table S2). Although a marked decrease in the synthesis of $9 \mathrm{C} \beta \mathrm{A} 10^{\prime} \mathrm{COL}$ was detected upon the introduction of trAtCCD8, we were unable to detect the synthesis of CL (fig. S6, A and B). Previous reports imply that CL was unstable $(31,37)$ and that $\mathrm{pH}$, solvent composition, and temperature can affect its chemical stability $(37,38)$. We added $200 \mathrm{mM}$ Hepes buffer ( $\mathrm{pH} 7.0)$ to the growth medium upon isopropyl $\beta$-D-1-thiogalactopyranoside (IPTG) induction (buffer volume/medium volume $=0.5$ ), which led to the detection of a tiny peak in chromatograms of both the cell pellets and the culture medium at $\mathrm{RT}=20.58 \mathrm{~min}$ with $\mathrm{m} / \mathrm{z}^{+}=$ 303.2, which agrees with that of CL (figs. S3E and S6, C and D). However, the yield of CL was low (fig. S6, C and D) and was not proportional to the decrease in the synthesis of 9C $\beta A 10^{\prime} \mathrm{COL}$. Thus, we tested different medium conditions for the detection of CL. Under the optimal fermentation conditions (XY medium; fig. S6, E to $G$ ), a distinguished peak with the maximum absorption at $269 \mathrm{~nm}$ and $\mathrm{m} / \mathrm{z}^{+}$consistent with those of CL at the same retention time as the putative CL was observed (Fig. 2, D and E, and fig. S3E) $(16,31)$.

\section{Synthesis of CLA in E. coli-S. cerevisiae coculture}

According to the pioneering in planta study, most of the canonical SLs are branched from CLA, which is synthesized from CL with the function of MAX1 (11). Although E. coli does not contain membranebound organelles, many plant cytochrome P450s have been functionally reconstitute in E. coli (39). However, further introduction of truncated MAX1 and cytochrome P450 reductase 1 from $A$. thaliana (40) (AtMAX1 and ATR1, respectively) in the CL-producing ECL-4 did not convert CL toward CLA (ECL-5; table S2 and fig. S8). Considering AtMAX1 is an endoplasmic reticulum (ER)-localized enzyme, the eukaryotic model species $S$. cerevisiae may be a more suitable organism to reconstitute the activity of the ER-localized CYP than E. coli (39). Since CL can be detected in both cell pellets and culture medium, it is possible to establish the synthesis of downstream SLs using an E. coli-S. cerevisiae coculture, with which CL is expected to be translocated from E. coli to yeast for further functionalization.

AtMAX1 and ATR1 were then introduced to S. cerevisiae on two low copy number plasmids and expressed downstream of PGK1 and TEF1 promoter, respectively (resulting strain: YSL-1; table S2). When the CL-producing E. coli strain (ECL-4) was cocultured with YSL-1, the peak of CL substantially decreased, and a new compound was detected in the organic extract of both cell pellets and medium under ultraviolet (UV) detection (Fig. 3A). The UV-visible (UV-VIS) spectrum of the peak had the maximum absorption at $271 \mathrm{~nm}$, with $[\mathrm{M}-\mathrm{H}]^{-}=331.1$ matching those of CLA (Fig. 3B and fig. S3F). High-resolution MS (HR-MS) analysis confirmed $[\mathrm{M}-\mathrm{H}]^{-}=331.1627$ consistent with that of CLA $\left(\mathrm{C}_{19} \mathrm{H}_{24} \mathrm{O}_{5}\right.$; fig. $\mathrm{S} 7 \mathrm{~A})$. We then tried to improve the CLA titer by adjusting the inoculum ratio between ECL-4 and YSL-1. We found that increasing the amount of yeast led to increased conversion of CL toward CLA (fig. S9). When the S. cerevisiae-E. coli inoculum ratio increased from 0.2 to 2 , the ratio between CLA and CL rose from 0.3 to 17.3. When the E. coli-yeast inoculum ratio was higher than 1, CLA production was not further improved, so we used the ratio in the subsequent experiments.

\section{Synthesis of OB through 4DO using CYP711 As in E. coli-S. cerevisiae coculture}

In rice, the two MAX1 homologs, OsCYP711A2 and OsCYP711A3, were identified to catalyze the conversion of CL to $4 \mathrm{DO}$ and $4 \mathrm{DO}$ to $\mathrm{OB}$, respectively $(22,24)$. To synthesize $4 \mathrm{DO}$ in the microbial system, we expressed Os900 (OsCYP711A2 gene) using a low copy number plasmid downstream of $P G K 1$ promoter in a yeast strain harboring ATR1 (YSL-2; table S2). When ECL-4 was cocultured with YSL-2, a peak with $\mathrm{m} / \mathrm{z}^{+}$at 331.1 (consistent with either 5DS or 4DO) was detected (Fig. 3C), which was further confirmed to be 4DO through comparison with the authentic 4DO and 5DS standards (Fig. $3 \mathrm{C}$ and figs. S3G and S10, A to C). The titer of $4 \mathrm{DO}$ in the consortium was $3.46 \pm 0.28 \mu \mathrm{g} /$ liter. In addition to $4 \mathrm{DO}$, we were also able to detect the synthesis of another new compound in comparison to the negative control (without OsCYP711A2) in the organic extract of the medium. The putative new compound showed $\mathrm{m} / \mathrm{z}^{-}$at 347.1 in the negative ion mode (figs. $\mathrm{S} 3 \mathrm{H}$ and $\mathrm{S} 10 \mathrm{~B}$ ) and is putatively 18-hydroxy-CLA (27). HR-MS analysis confirmed that $[\mathrm{M}-\mathrm{H}]^{-}=347.1521$, and the fragmentation patterns are both consistent with those of 18-hydroxy-CLA previously reported $\left(\mathrm{C}_{19} \mathrm{H}_{24} \mathrm{O}_{6}\right.$; fig. S7B) (27).

Furthermore, we introduced Os1400 (gene of OsCYP711A3) to YSL-2 on a low-copy plasmid driven by GPD promoter (YSL-3; table S2). As expected, compared to the ECL-4/YSL-3N (YSL-3N is equivalent to YSL2 but harboring an additional empty vector; table S2) coculture, the ECL-4/YSL-3 coculture synthesized substantially less $4 \mathrm{DO}$ and $\mathrm{OB}(0.75 \pm 0.01 \mu \mathrm{g} /$ liter) (identified and quantified by using the authentic OB standard; Fig. 3D and figs. S3I and S11). Different from $4 \mathrm{DO}$ detected in both pellets and medium, OB mostly in the medium indicates that most $\mathrm{OB}$ synthesized was exported into the medium. The successful reconstitution of $\mathrm{OB}$ synthesis from CL in the microbial consortium using OsCYP711A2 and OsCYP711A3 confirmed the previously proposed synthetic pathway of OB in rice (24).

\section{Synthesis of OB using CYP722C in E. coli-S. cerevisiae coculture} Previous investigations confirmed that VuCYP722C from cowpea can directly convert CLA into OB and its diastereomer ent- $2^{\prime}$-epi$\mathrm{OB}$ by in vitro experiments (27). To establish this OB biosynthetic route in the microbial system, we introduced VuCYP722C to YSL-1 on a low copy number plasmid downstream of the GPD promoter (YSL-4; table S2), then cocultured YSL-4 with the CL-producing ECL-4. The ECL-4/YSL-4 coculture produced much less CLA than the ECL- $4 / \mathrm{YSL}-4 / 5 \mathrm{~N}$ coculture (YSL- $4 / 5 \mathrm{~N}$ is equivalent to YSL- 1 but harboring an additional empty vector; table S2 and fig. S12A), and synthesized $19.36 \pm 5.20 \mu \mathrm{g} /$ liter OB (Fig. 3D and fig. S12C). We were also able to detect the putative 18-hydroxy-CLA in the medium of the ECL-4/YSL-4 coculture (fig. S12B). Our results confirmed that $\mathrm{OB}$ can be generated through two different routes using different set of CYPs.

\section{Synthesis of 5DS using CYP722C in E. coli-S. cerevisiae coculture} GaCYP722C from cotton was reported to be responsible for the conversion of CLA into 5DS through an in vitro investigation (28). To synthesize 5DS in microbial consortium, we introduced the GaCYP722C gene into the CLA-producing yeast on a low copy number plasmid downstream of GPD promoter (YSL-5; table S2). When YSL-5 was cocultured with the CL-producing ECL-4, we detected 5DS (6.65 $\pm 1.71 \mu \mathrm{g} /$ liter) (5DS was identified and quantified 

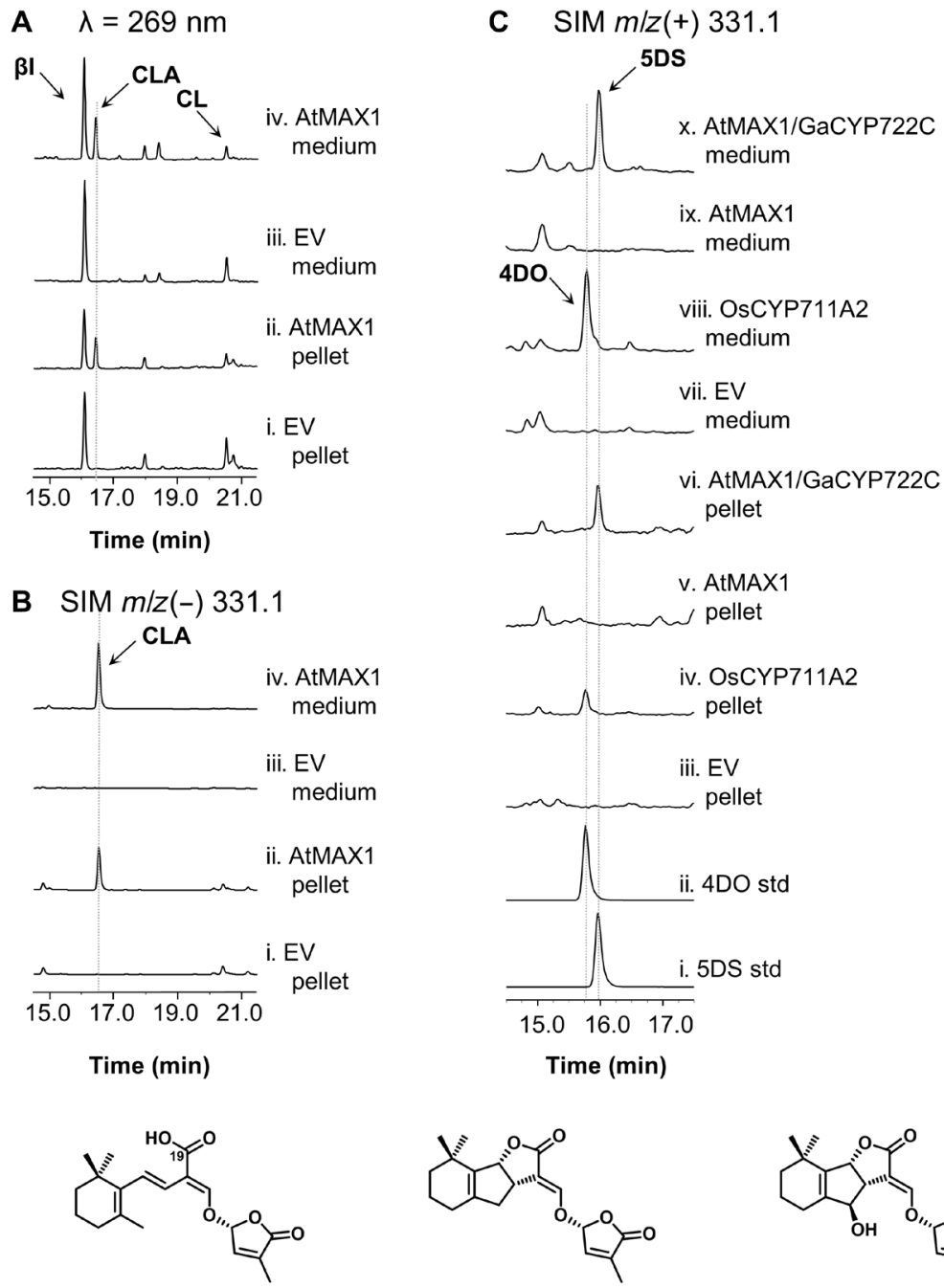

Carlactonoic acid, CLA 4-Deoxyorobanchol, 4DO

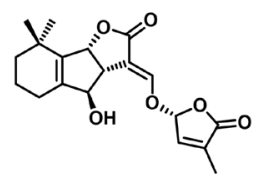

Orobanchol, OB
D $\quad \operatorname{SIM~} m / z(+) 347.1$
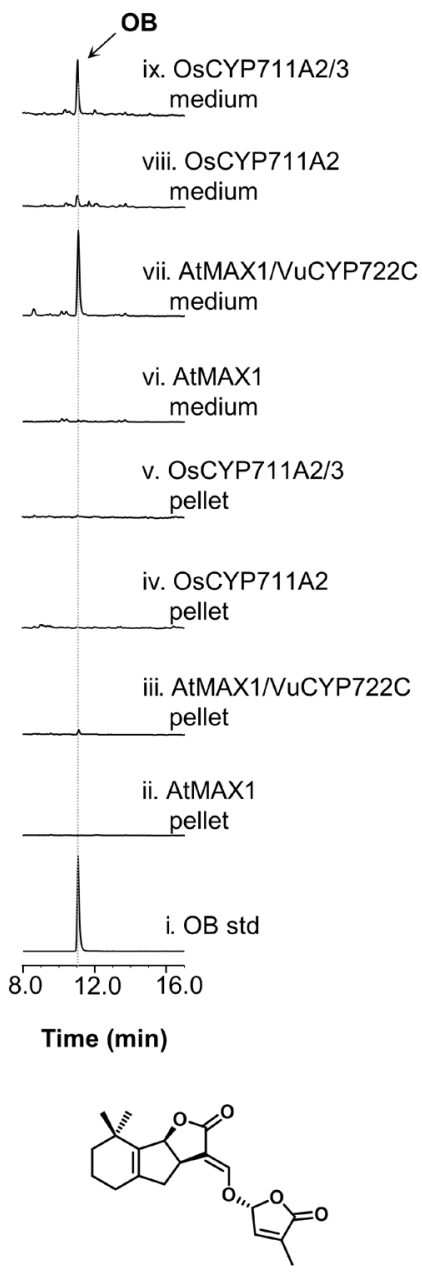

5-Deoxystrigol, 5DS

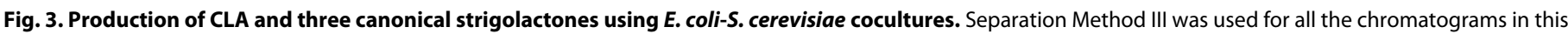

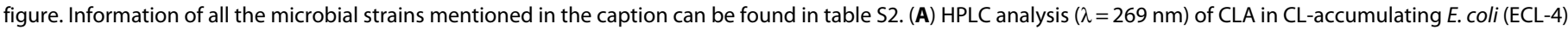

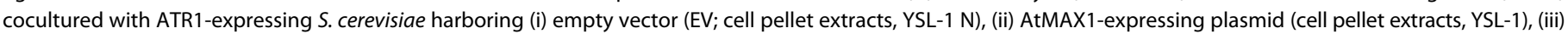

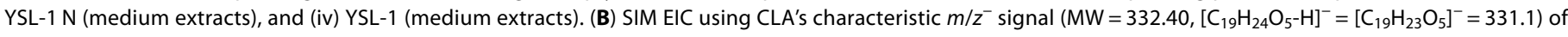

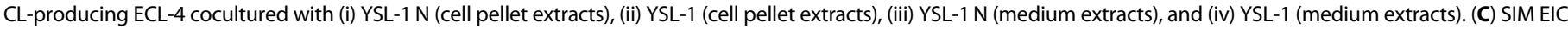

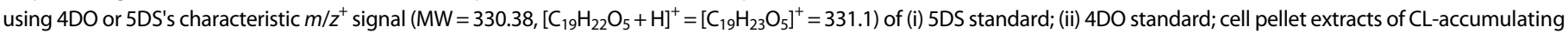

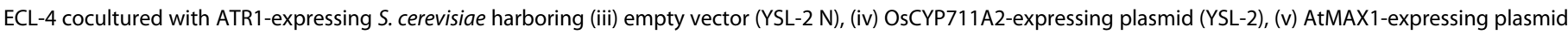

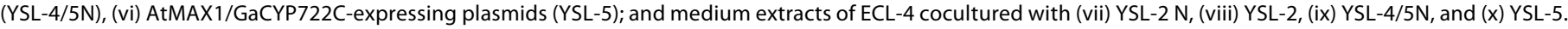

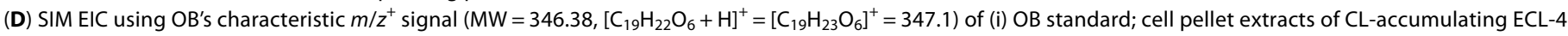

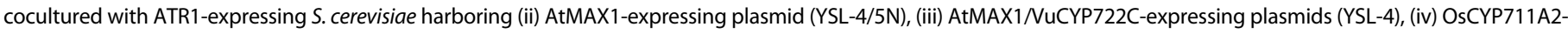

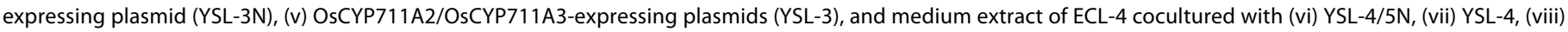
YSL-3N, and (ix) YSL-3. All traces are representative of at least three biological replicates for each engineered E. coli-S. cerevisiae consortium.

by using the authentic standard; Fig. 3C and figs. S3J and S13C). Different from OB, 5DS was detected in the extract of both medium and cell pellet, which aligns with the lower hydrophilicity of 5DS than OB (fig. S13). Only a small quantity of CLA was detected (fig. S13A), suggesting the high efficiency of GaCYP722C. The putative 18-hydroxy-CLA was also detected in the medium of the 5DSproducing consortium (fig. S13B). This peak exists in the medium of all the canonical SL-producing consortiums we constructed, which supports the hypothesis that 18 -hydroxy-CLA is a common intermediate in canonical SLs biosynthesis.

\section{Functional mapping of various CYP722Cs}

CYP722C genes are widely distributed in flowering plants. GaCYP722C share $65 \%$ amino acid identity with VuCYP722C, yet they catalyze different reactions. It is intriguing to investigate whether the enzymatic function of homologous proteins is conserved across different plant species. The successful functional reconstitutions of GaCYP722C and VuCYP722C in the microbial consortium hint the potential of using this biosynthetic platform to establish a sequence-function correlation of CYP722Cs, which will enable predicting the function of unknown CYP722Cs. We used GaCYP722C protein sequence as 
a query, performed BLASTp search, and selected a total of 28 CYP722C sequences from different plant species including dicotyledons and monocotyledons (table S4). The SL profile of some of these plant species has been examined. For example, birdsfoot trefoil (Lotus japonicus) (2) and woodland strawberry (Fragaria vesca) were reported to produce 5DS (37), while cowpea (Vigna unguiculata) $(26,41)$, red bell pepper (Capsicum annuum) (25), and red clover (Trifolium pratense) were reported to produce $\mathrm{OB}$ without involving $4 \mathrm{DO}(25,41)$. Tomato (Solanum lycopersicum) produces OB (42). We also included two CYP722Cs with the functions to be confirmed [SlCYP722C (27) and LjCYP722C (43)] and CYP722A and CYP722B sequences as the outgroup (Fig. 4).

Phylogenetic analysis indicated that CYP722C subfamily can be divided into two groups (Fig. 4 and fig. S15): group I and group II. The characterized OB-producing VuCYP722C and SlCYP722C are members of group I. The speculative 5DS synthase LjCYP722C and characterized 5DS-producing GaCYP722C are members of group II. To examine whether we can simply predict the function on the basis of the phylogenetic analysis, we synthesized eight CYP722C genes (table S3) from different branches and screened their functions by introducing each gene to the CLA-producing microbial consortium on a low copy number plasmid regulated by GPD promoter (table S2). Among the eight CYP722C genes we examined, all the CYP722Cs from group I [S. lycopersicum, C. annuum, T. pratense, Glycine max (Soybean), Citrus sinensis (Sweet orange), and Vitis vinifera (Grape)] indeed converted CLA to OB (Fig. 4 and fig. S16A), while the two CYP722Cs from group II (F. vesca and L. japonicus) synthesized 5DS from CLA (Fig. 4 and fig. S16B). We also examined the function of OsCYP722B (Oryza sativa) and SbCYP722B (Sorghum bicolor) using the CLA-producing consortium and did not detect any conversion of CLA or synthesis of 5DS or OB (table S2 and fig. S14). The CYP722C characterization results are consistent with the previously reported SL profiles from the corresponding plants $(2,25,26,37,41,42)$ and suggest that sweet orange and grape are capable of synthesizing $\mathrm{OB}$, although their SL profiles have not been reported.

The SL-profile of most plants is not reported. The phylogenic analyses and functional characterization of CYP722Cs from various plant species in the microbial system imply a sequence-function correlation, which can be used to predict the SL synthetic capacity of the corresponding plants. If a plant encodes a group I CYP722C, then likely it is able to produce $O$-type SLs, while if a group II CYP722C is present, this plant should own the capability to synthesize $S$-type SLs.

\section{Metabolic engineering to improve 5DS production}

After demonstrating microbial biosynthesis of various SLs, we used metabolic engineering to improve product titer, which should facilitate future gene characterization efforts. We chose 5DS as the model SL. We first improved the CL-producing E. coli (ECL-4). In this strain, the building blocks of CL (isopentenyl diphosphate and dimethylallyl diphosphate) were supplied by an endogenous, nonengineered methylerythritol phosphate (MEP) pathway. Biosynthesis of many isoprenoids was found in prior studies to be limited by this pathway (44-47), possibly due to that $E$. coli evolved to regulate this pathway to have low flux because of weak demand of isoprenoids by the cell. To up-regulate the MEP pathway, we overexpressed its first enzyme, 1-deoxy-D-xylulose-5-phosphate synthase (EcDXS; coded by $d x s$ ). Since $d x s$ is in the same operon in the $E$. coli genome with ispA (a gene also involved in the CL biosynthetic pathway), the dxs-ispA operon including its native promoter and terminator was inserted into $p A C$-BETAipi. IspA encodes farnesyl diphosphate synthase (EcEPPS) and would also be up-regulated in the new strain (ECL-6; table S2). When ECL-4 was replaced by ECL-6 in the

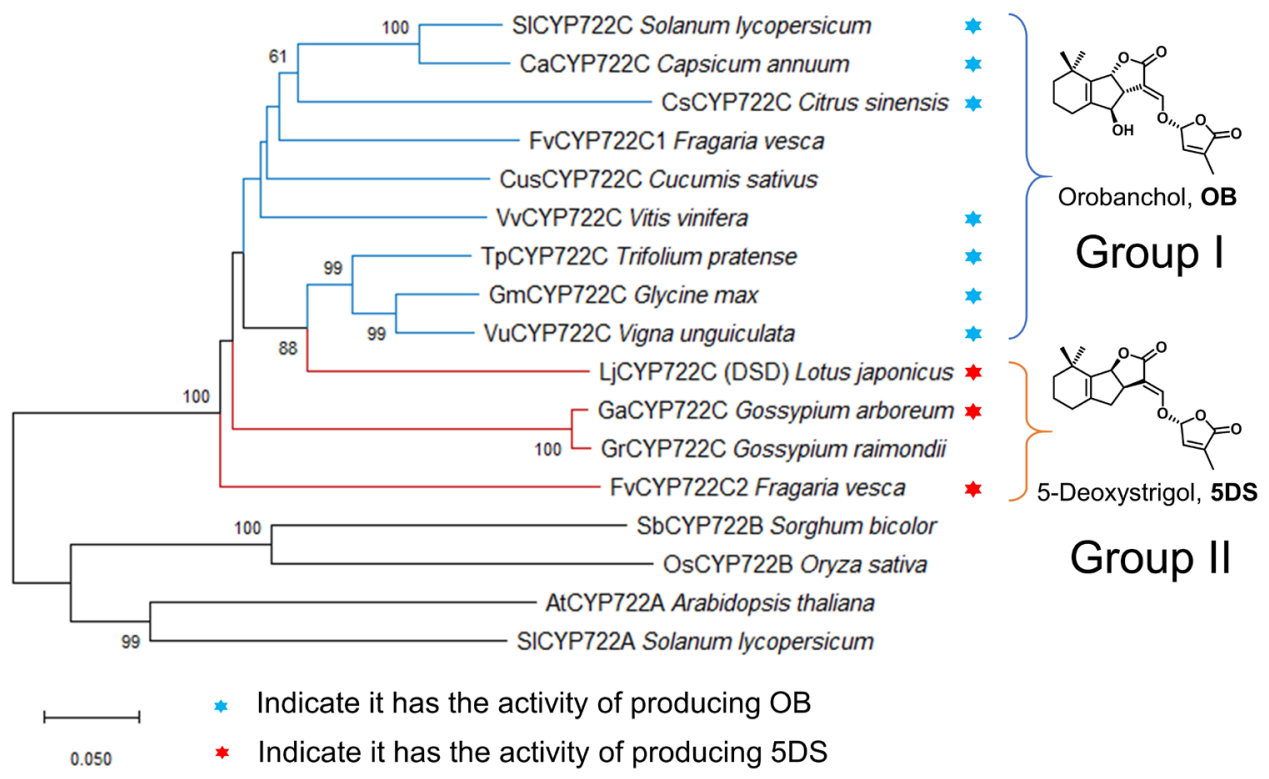

Fig. 4. Phylogenetic analysis of CYP722C and the functional mapping. Phylogenetic analyses were conducted in MEGA X by using ClustalW for multiple sequence alignment and the neighbor-joining method. The parameters are set as follows: bootstrap test (1000 replicates), p-distance mode, and complete deletion. The numbers next to branches indicate bootstrap values ( $>60 \%$ are shown). This analysis involved 17 amino acid sequences, including CYP722A and CYP722B sequences to root the tree. The accession numbers of proteins are listed in table S4. The asterisk means that these enzymes have been tested in this study, blue asterisk indicates OB-producing activity, and red asterisk indicates 5DS producing activity. 
5DS-producing coculture (ECL4/YSL-5), the pool size of CL was increased by $150 \%$ (Fig. 5A), but the 5DS titer was not increased (Fig. 5B). Since the increase in the CL pool size could also be due to reduced consumption of CL, we analyzed a by-product $(\beta I)$ of the CL biosynthetic pathway. Because there was no reaction known to consume $\beta I$ in this culture, its titer can be used as an indicator of the CL production rate. The $\beta$ I titer of the ECL-6/YSL- 5 coculture was $330 \%$ (Fig. 5C) higher than that of the ECL-4/YSL-5 coculture, suggesting that the overexpression of $d x s$ and isp $A$ indeed improved the CL production in the coculture and that it is needed to improve the conversion of CL into 5DS in YSL-5.

Since the SL biosynthesis is less understood, it was more difficult to pinpoint the rate-limiting step. We then decided to increase the concentration of all the enzymes involved in functionalizing CL by enlarging the population of YSL-5. The yeast growth was limited by acetate supply (acetate concentration constantly below detection limit, $0.1 \mathrm{~g} /$ liter, during the coculture; acetate was the main carbon source of the yeast and was produced by E. coli), so ethanol was added as a supplement carbon source for $S$. cerevisiae. Ethanol can be converted into acetate in the yeast and cannot be metabolized by E. coli (48). The yeast growth was indeed improved by supplementing ethanol (4 g/liter; fig. S17), and almost no CL was accumulated under this condition (Fig. 5A). 5DS titer was increased by $220 \%$ (to $47.3 \mu \mathrm{g} /$ liter; Fig. $5 \mathrm{~B}$ ) compared with the coculture without the ethanol addition.

In addition, OsD27 in ESL-6 was replaced with six D27 variants from different plant species individually (Oryza nivara, Oryza rufipogon, Oryza meridionalis, Triticum aestivum, Zea mays, and Populus trichocarpa). Although none of these replacements further increased the 5DS production (fig. S18), these exercises quantitatively compare the activities of variants of SL biosynthetic enzymes from different plant species, which may shed light on evolution of SL biosynthesis (elaborated below).

\section{DISCUSSION}

The biosynthetic pathway from AT $\beta C$ to $C L$ is conserved in most land plants $(49,50)$; however, the routes for the conversion of CL into canonical SLs can be distinct among different plant species, even when they produce the same SLs (50). A microbial platform of producing CL, CLA, and the downstream pathway intermediates may make the characterization of the unknown pathways much easier. Our study indicates that it is highly challenging to establish the biosynthesis of CL in yeast but possible in E. coli. Through using the $E$. coli-yeast coculture strategy, we established the biosynthesis of CL in E. coli and then various SLs in the E. coli-yeast consortium. The SL-producing microbial consortium, although currently with low SL titer (at the level of tens of microgram per liter), provides a handy platform for the functional characterization of SL biosynthetic enzymes, as in the proof-of-concept experiment on CYP722Cs (fig. S16). Through simply introducing the CYPs of interest to the yeast strain of the corresponding SL-producing microbial consortium (for CYP722Cs, CLA-producing consortium), we were able to identify the function of CYP722Cs within 2 weeks upon arrival of the synthetic genes. This approach does not need to supply synthetic CLA, which is chemically unstable and commercially unavailable. The functions of the CYP722Cs identified by the microbial system were consistent with the reported SL profiles of the corresponding plants.

SLs exhibit extremely low abundance in nature (up to $70 \mathrm{pg}$ of OB per plant detected in the roots of red clover seedlings) $(51,52)$, making isolation of SLs from plant materials to be laborious and costly. Because of the structural complexity and instability of SLs, the chemical synthesis of SLs is laborious and expensive to be an economic SL supply strategy for the SL-based agricultural applications $(4,9,53)$. Chemical synthesis has been useful in SL-related research through providing some synthetic SL analogs (4), such as GR24. However, synthetic analogs are generally less active than natural SLs (54) and generally in racemic mixtures, while different isomers exhibit different activities (55). For example, the commonly used synthetic SL analog GR24 is 100-fold less potent at stimulation of O. minor germination than natural SLs (56), and (+)-GR24 is 100 times more active than (-)-GR24 as a branching factor (57). Unfortunately, many conclusions on the biological effects of SLs are obtained using racemic GR24 $(6,8,58)$. In addition, SLs of subtle structural differences may exhibit different bioactivities $(49,59)$. For example, different SLs have been found to exhibit distinct efficiencies toward

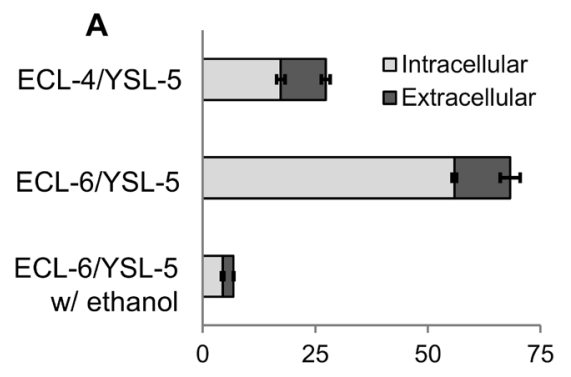

Titer of $\mathrm{CL}$ at 72 hours (peak area[mAU]/ml)
B

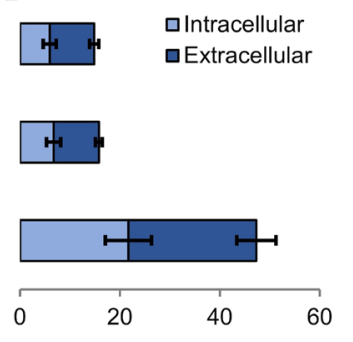

Titer of 5DS at 72 hours $(\mu \mathrm{g} /$ liter $)$
C
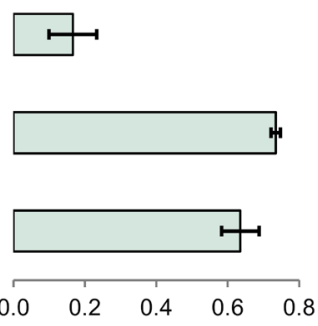

Titer of $\beta \mathbf{I}$ at 72 hours $(\mathrm{mg} /$ liter $)$

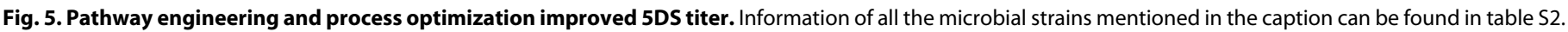

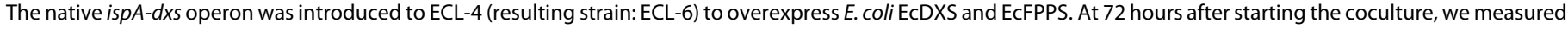

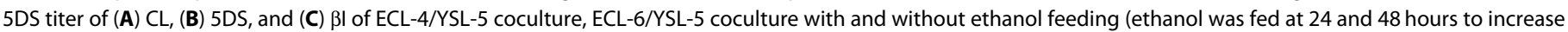

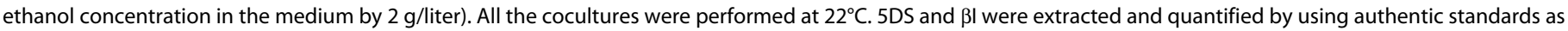

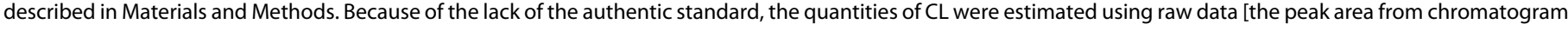

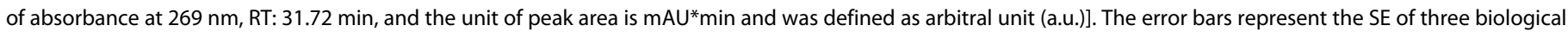
replicates. 
shoot branching inhibition in plants and hyphal branching activity in arbuscular mycorrhizal fungi (AMF) (49). The limited access to natural SLs is hindering comprehensive investigations on the structure-activity correlation of this group of phytohormones in plant. The development of efficient microbial bioproduction of various natural SLs will advance these investigations and agricultural applications.

Much remains to be investigated into the biochemistry and evolution of CYP722Cs. Consistent with the pioneering in planta and biochemical characterizations of CYP722Cs $(27,28)$, our study also implies that CYP722Cs are divided into two major groups, one group converts CLA to OB (group I) and the other one synthesizes 5DS (group II). Both types of CYP722Cs are believed to synthesize OB or 5 DS through first catalyzing 18-hydroxylation of CLA $(27,28)$. Group I CYP722C further catalyzes the oxidation of the alcohol at $\mathrm{C} 18$ to aldehyde that triggers nucleophilic attack to afford B ring and $\mathrm{C}$ ring closures for the synthesis of $\mathrm{OB}$ (the oxygen atom is retained at C18). This process would not produce 4DO (fig. S19) (27). On the other hand, group II CYP722C does not catalyze further oxidation of 18-hydroxyl; it may protonate the 18-hydroxyl to serve as a leaving group to afford the synthesis of 5DS (fig. S19) (28). In the functional identification of various CYP722Cs, we did not detect the synthesis of 4DO in the CYP722C-expressing SL-producing microbial consortium. Further investigations into CYP722Cs from other plants may help answer whether there is a $4 \mathrm{DO}$-synthesizing CYP722C. It is also interesting to understand how CYP722Cs evolve to control the $\mathrm{C} 18$ oxidation and stereospecificity of ring closing.

A compound detected in the 4DO-producing microbial consortium (ECL-4/YSL-2) may putatively be 18-hydroxy-CLA (fig. S10B). The synthesis of 4DO by OsCYP711A2 in ECL-4/YSL-2 is likely through a similar mechanism as GaCYP722C except that OsCYP711A2 catalyzes one more oxidation at $\mathrm{C} 19$ (to carboxylic acid) in addition to the C18 oxidation (to alcohol; fig. S19) (24). We did not detect any $5 \mathrm{DS}$ or $\mathrm{OB}$ in the $4 \mathrm{DO}$-producing microbial consortium using OsCYP711A2 (ECL-4/YSL-2). Further biochemical investigations are needed to understand how CYP711As that convert CL to CLA were evolved to gain $\mathrm{C} 18$ hydroxylation ability and to catalyze the $\mathrm{B}$ ring and $\mathrm{C}$ ring closures with strict stereospecificity. The microbial platform we have built should be able to expedite enzyme characterizations to answer these questions.

In addition, the establishment of deoxy-SL (4DO and 5DS) producing microbial consortium may provide a handy platform for the discovery of the downstream tailoring enzymes in SL biosynthesis. Hydroxylation on SLs can alter the biological activities. For example, Striga-susceptible maize cultivar only produces 5DS, while mainly sorgomol is detected in the resistant cultivar (60); Sorghum produces both 5DS and sorgomol and can convert 5DS to sorgomol (26). Recently, CYP728B35 encoded by Sorghum has been suggested to be the sorgomol synthase catalyzing the hydroxylation of 5DS to afford the synthesis of sorgomol (61). In addition to Sorghum, strigolproducing cotton converts 5DS to strigol and strigyl acetate (26). The enzymes involved in these oxidations of SLs remain to be characterized.

Moreover, the biosynthesis of noncanonical SLs remains largely unknown. In Arabidopsis, CLA can be methylated to form MeCLA by an unknown methyltransferase (11). Recently, lateral branching oxidoreductase, a 2-oxoglutarate- and $\mathrm{Fe}^{\mathrm{II}}$-dependent dioxygenase, was suggested to catalyze the hydroxylation of MeCLA to hydroxymethylcarlactonoate (1'-HO-MeCLA) $(62,63)$, which may be the precursors of many noncanonical SLs such as avenaol (37). In addition to MeCLA and 1'-HO-MeCLA, diverse noncanonical SLs, including hydroxyl CL (3-HO-CL, 4-HO-CL, and 16-HO-CL) and the corresponding hydroxyl CLA derivatives, were also detected in Arabidopsis (63), with little known about the enzymes involved in the formation of these structures. L. japonicus can produce noncanonical SL lotuslactone (15), and a 2-oxoglutarate-dependent dioxygenase (2-OGD) (named $L L D$ ) gene was proposed to be involved in the biosynthesis of lotuslactone (43), yet the catalytic function of LLD was not characterized. The CL/CLA-producing microbial consortium may also provide a convenient way to functionally identify and discover the enzymatic mechanism in the biosynthesis of various SL structures, both canonical and noncanonical.

Another intriguing yet mysterious question to be answered is the origin and evolutionary history of SL biosynthesis and perception. SLs have been reported to be present in several bryophytes and green algae $(29,30,64)$, which do not always encode the full set of SL biosynthetic enzymes as described above $(29,30)$. These facts spur debate about the origin and evolution of SL biosynthesis and perception $(29,30)$. The moss Physcomitrella patens encodes one set of D27, CCD7, and CCD8, but no MAX1 analog, which is consistent with previous investigation that $P$. patens only synthesizes $\operatorname{CL}(29,37)$. The activities of $P$. patens CCD7 (PpCCD7) and CCD8 (PpCCD8) toward the synthesis of CL have been validated through in vitro reconstitution (65). On the other hand, CCD8 and MAX1 analogs are absent from the genome of liverworts Marchantia polymorpha $(29,66)$. In this study, the activity of P. patens D27 (PpD27), PpCCD8, and $M$. polymorpha CCD7 (MpCCD7) were examined in the CL-producing ECL-6 through replacing the corresponding ortholog (e.g., PpD27 replacing OsD27; fig. S20). Replacing OsD27 with $\mathrm{PpD} 27$ retained the synthesis of CL, while replacing AtCCD7 or AtCCD8 with MpCCD7 or PpCCD8 completely abolished CL production (fig. S20). The inconsistency in the activity of PpCCD8 between our study and previous investigations might be due to different $\mathrm{N}$ terminus truncation or codon optimization method (we truncated four more amino acids to be consistent with trAtCCD8; table S6). MpCCD7 does not encode a chloroplast transfer peptide and is more than $10 \%$ longer in length than regular CCD7s, and the activity of MpCCD7 might be different from regular CCD7s and requires further investigations (e.g., assay with more substrates than 9C $\beta C$ ). Furthermore, since MAX1 and CYP722 analogs are universally present in flowering plants yet generally absent from primitive plants $(29,67)$, we also examined the activity of some MAX1 and CYP722 analogs from Sphagnum fallax (moss, SfMAX1, 46.1\% identity to AtMAX1; SfCYP722C, 36.2\% identity to GaCYP722C) and Klebsormidium nitens (green algae, four MAX1 orthologs, KnMAX1a-1d, 27.9 to $31.2 \%$ identity to AtMAX1) in the CL-producing consortium (66). Unfortunately, none of these CYPs examined converted CL to CLA or CLA to 5DS or OB in the microbial consortia (fig. S21), suggesting different functions from AtMAX1 or group I or II CYP722Cs. These species may produce SL-like compounds that have not been found yet, with distinct biosynthetic pathway from seed plants, which need to be characterized. Comprehensive investigations into the putative SL biosynthetic enzymes from primitive plants are necessary to fully elucidate the SL biosynthesis from these plants as well as the origin and evolution of SL biosynthesis.

The current titer of 5DS ( $\sim 50 \mu \mathrm{g} /$ liter $)$ needs to be further improved to isolate pathway intermediates of SL biosynthesis for structural verification (e.g., 18-hydroxy-CLA) and mechanistic investigation of SL biosynthetic enzymes (e.g., CYP722Cs). We engineered an 
E. coli strain that could produce AT $\beta C$ ( $~ 200 \mathrm{mg} /$ liter $)$ by using the recently developed isopentenol utilization pathway (68). But when we introduced the CL-synthesizing pathway into this strain, much less CL was produced compared with using the engineered MEP pathway. A future study should investigate how to efficiently convert AT $\beta C$ into CL in high AT $\beta C$-producing strains. A special attention should be paid to D27, which is the first enzyme in this segment and may control its flux. D27s from different species could be screened, and the best natural D27 could be further improved by directed evolution.

Furthermore, although our study demonstrates that microbial consortium is a promising mechanism for the supply of various SLs, single strain-based SL-producing microorganism is still desired because of the easier manipulation during fermentation. To reconstruct SL-producing E. coli strains, further engineering on the functional reconstitution of multiple CYPs (i.e., CYP722Cs or CYP711As) in $E$. coli is critical. On the other hand, mechanistic investigation into the failed reconstitution of D27 and CCDs in yeast may enable the rational design of microenvironment to functionally reconstitute these plastid-localized enzymes in yeast.

As a conclusion, in this study, we have successfully reconstituted the biosynthesis of CL, CLA, and three canonical SLs (4DO, 5DS, and $\mathrm{OB}$ ) in E. coli-yeast microbial consortia. Our study highlights the usefulness of the microbial platform as a convenient and rapid way to characterize the function of SL biosynthetic enzymes.

\section{MATERIALS AND METHODS}

\section{Chemicals and general culture conditions}

$( \pm) 5$-deoxy-strigol (purity $>98 \%$ ) and $( \pm)$-OB were purchased from Strigolab (Italy). ( \pm ) $\mathrm{DO}$ [also named as $( \pm)-2^{\prime}$-epi-5DS] were acquired from ChemPep Inc. (USA). $\beta$ I is purchased from Thermo Fisher Scientific (USA). AT $\beta C$ and 9 C $\beta C$ were purchased from Sigma-Aldrich Co. (USA). The chemically competent E. coli strain TOP10 (Life Technologies) was used for DNA manipulation and amplification and was grown at $37^{\circ} \mathrm{C}$ in lysogeny broth (LB) medium (Thermo Fisher Scientific) supplemented with appropriate amount of antibiotics [ampicillin $(100 \mu \mathrm{g} / \mathrm{ml}$; Thermo Fisher Scientific), kanamycin $(50 \mu \mathrm{g} / \mathrm{ml}$; Thermo Fisher Scientific), chloramphenicol $(25 \mu \mathrm{g} / \mathrm{ml}$; Thermo Fisher Scientific), and/or spectinomycin $(50 \mu \mathrm{g} / \mathrm{ml}$; Sigma-Aldrich)] for plasmid maintenance. For protein expression and CL production, we used chemically competent $E$. coli strain BL21(DE3) (Novagen) and LB (or XY medium). XY medium contains $\mathrm{KH}_{2} \mathrm{PO}_{4}(13.3 \mathrm{~g} /$ liter $),\left(\mathrm{NH}_{4}\right)_{2} \mathrm{HPO}_{4}(4 \mathrm{~g} /$ liter $)$, citric acid (1.7 g/liter), $\mathrm{CoCl}_{2}$ (0.0025 g/liter), $\mathrm{MnCl}_{2}$ (0.015 g/liter), $\mathrm{CuCl}_{2}$ (0.0015 g/liter), $\mathrm{H}_{3} \mathrm{BO}_{3}(0.003 \mathrm{~g} /$ liter $), \mathrm{Na}_{2} \mathrm{MoO}_{4}(0.0025 \mathrm{~g} /$ liter $)$, $\mathrm{Zn}\left(\mathrm{CH}_{3} \mathrm{COO}\right)_{2}(0.008 \mathrm{~g} /$ liter $), \mathrm{Fe}(\mathrm{III})$ citrate $(0.06 \mathrm{~g} /$ liter $)$, thiamine (0.0045 g/liter), $\mathrm{MgSO}_{4}(1.3 \mathrm{~g} /$ liter $)$, yeast extract (5 g/liter), and xylose ( $40 \mathrm{~g} /$ liter $), \mathrm{pH}$ 7.0. In the first stage of the coculture fermentation, yeast strains were cultured at $28^{\circ} \mathrm{C}$ in complex yeast extract, peptone, and dextrose (YPD; all components from $\mathrm{BD}$ Biosciences) medium or synthetic dropout (SD) medium containing yeast nitrogen base (1.7 g/liter) without amino acids (BD Biosciences), ammonium sulfate ( $5 \mathrm{~g} /$ liter; Thermo Fisher Scientific), dextrose ( $20 \mathrm{~g} /$ liter), and suitable SD mixture at the concentrations specified by the manufacturer (Clontech). XY medium was used in the second stage of the coculture fermentation. Unless specified, all the chemicals used in this study were purchased from Thermo Fisher Scientific or Sigma-Aldrich Co.

\section{General techniques for DNA manipulation}

Plasmid DNA was prepared using the EconoSpin columns (Epoch Life Science) according to the manufacturer's protocols. Polymerase chain reactions (PCRs) were performed using Q5 DNA polymerase [New England Biolabs (NEB)] and Expand High Fidelity PCR System (Roche Life Science) according to the manufacturer's protocols. PCR products were purified by the Zymoclean Gel DNA Recovery Kit (Zymo Research). All DNA constructs were confirmed through DNA sequencing by Source BioScience (LA, USA) or BioBasic (Singapore). Restriction enzymes (NEB) and T4 ligase (NEB) were used to produce and ligate the DNA fragments, respectively. BP Clonase II Enzyme Mix, Gateway pDONR221 Vector and LR Clonase II Enzyme Mix (Invitrogen), and the S. cerevisiae Advanced Gateway Destination Vector Kit (Addgene) were used to perform Gateway cloning (69). Using this method, the yeast expression cassette vectors were constructed. Plasmids were introduced into yeast cells using the Frozen-EZ Yeast Transformation II Kit (Zymo Research). Gibson one-pot, isothermal DNA assembly was conducted at $10-\mu$ l scale by incubating T5 exonuclease (NEB), Phusion polymerase (NEB), Taq ligase (NEB), and 50 ng of each DNA fragment at $50^{\circ} \mathrm{C}$ for 1 hour to assemble multiple DNA fragments into one circular plasmid (70). Integrated yeast strains were constructed through homologous recombination and DNA assembly (71). Plasmids and microbial strains used in this study are listed in tables S1 and S2, respectively. DNA oligonucleotides were synthesized by Integrated DNA Technologies (IDT) and Life Technologies. The plant gene sequences were codon-optimized for expression in $S$. cerevisiae and synthesized by IDT (Coralville, IA) and Twist Bioscience (San Francisco, CA). DNA sequences of genes involved in this work are listed in table S6.

For the construction of $E$. coli expression vectors, the truncated AtCCD7 gene was amplified by PCR and cloned into the pCDFDuet-1 plasmid (Novagen) using Nco I and Not I to yield the plasmid pCDFDuet-trAtCCD7. The OsD27 gene was amplified by PCR, digested by Nde I and Avr II, and ligated into accordingly digested pCDFDuet-trAtCCD7, yielding the plasmid pCDFDuet-trAtCCD7OsD27. Using the same strategy, the other six D27 homologous genes were cloned into the pCDFDuet-trAtCCD7 vector, respectively, yielding the corresponding coexpression plasmid. The OsD27, truncated OsD27, and AtD27gene was PCR-amplified, digested by Nde I and Avr II, and ligated into accordingly digested pCDFDuet-1, yielding the plasmid pCDFDuet-OsD27, pCDFDuet-trOsD27, and pCDFDuet-AtD27. The truncated AtCCD8 gene was amplified by PCR and cloned into pET21a using Gibson assembly, yielding pET21a-trAtCCD8. The truncated PpCCD8 was cloned into pET21a, yielding pET21a-trPpCCD8. For the construction of yeast expression cassettes, NADPH-P450 reductase and each individual P450 gene were constructed using Gateway cloning as described previously. The plasmid of pAC-BETAipi-ispA/dxs was constructed by using a Gibson assembly kit (Gibson Assembly Master Mix, NEB). The insert was amplified from genomic DNA of E. coli BL21(DE3) by using colony PCR and assembled with the backbone of pAC-BETAipi generated by using PCR.

\section{Culture conditions for $E$. coli-based CL precursors production}

For the in vivo production of 9C $\beta C, E$. coli BL21(DE3) was transformed with pAC-BETAipi (Addgene) and pCDFDuet-OsD27, generating E. coli ECL-2. For 9C $\beta A 10$ 'COL production, E. coli 
BL21(DE3) was transformed with pAC-BETAipi (Addgene) and pCDFDuet-OsD27-trAtCCD7 to generate E. coli ECL-3. Then, the yellow colonies were picked and grown in LB with chloramphenicol $(25 \mu \mathrm{g} / \mathrm{ml})$ and spectinomycin $(50 \mu \mathrm{g} / \mathrm{ml})$ at $37^{\circ} \mathrm{C}$, overnight. Fifty microliters of the overnight culture was then used to inoculate $5 \mathrm{ml}$ of fresh LB with the corresponding antibiotics with a starting optical density at $600 \mathrm{~nm}\left(\mathrm{OD}_{600}\right)$ at $\sim 0.05$ and cultured at $37^{\circ} \mathrm{C}$ and $220 \mathrm{rpm}$ in the $100-\mathrm{ml}$ Erlenmeyer flask. When $\mathrm{OD}_{600}$ reached $\sim 0.6$, IPTG was added to make the final concentration at $0.2 \mathrm{mM}$, with ferrous sulfate supplemented at the same time (final concentration at $10 \mathrm{mg} / \mathrm{liter}$ ). Then, the cultures were cultivated at $22^{\circ} \mathrm{C}$ and $220 \mathrm{rpm}$ for 72 hours.

\section{Culture conditions for $E$. coli-yeast consortium-based SL production}

For the in vivo production of SLs, E. coli BL21(DE3) was cotransformed with the plasmids pAC-BETAipi (Addgene), pCDFDuetOsD27-trAtCCD7, and pET21a-trAtCCD8, generating E. coli ECL-4. Single yellow colony was then picked and grown overnight at $37^{\circ} \mathrm{C}$ in $1 \mathrm{ml}$ of LB supplemented with ampicillin $(100 \mu \mathrm{g} / \mathrm{ml})$, chloramphenicol $(25 \mu \mathrm{g} / \mathrm{ml})$, and spectinomycin $(50 \mu \mathrm{g} / \mathrm{ml})$. Fifty microliters of the overnight culture was then used to inoculate $5 \mathrm{ml}$ of fresh LB with the corresponding antibiotics with a starting $\mathrm{OD}_{600}$ at $\sim 0.05$ and cultured at $37^{\circ} \mathrm{C}$ and $220 \mathrm{rpm}$ in the $100-\mathrm{ml}$ Erlenmeyer flask. When $\mathrm{OD}_{600}$ reached $\sim 0.6$, IPTG was added with the final concentration at $0.2 \mathrm{mM}$, with ferrous sulfate supplemented at the same time (final concentration at $10 \mathrm{mg} /$ liter). Then, the cultures were incubated at $22^{\circ} \mathrm{C}$ and $220 \mathrm{rpm}$ for 15 hours.

In parallel to preparing the $E$. coli culture, single colony of each yeast strain harboring the corresponding cytochrome P450-expression constructs was used to inoculate an appropriate SD medium considering the auxotrophic markers for maintaining the plasmid(s). The seed culture was incubated at $28^{\circ} \mathrm{C}$ and $220 \mathrm{rpm}$ overnight. One hundred microliters of the overnight grown seed culture was used to inoculate $5 \mathrm{ml}$ of the corresponding SD medium in a $100-\mathrm{ml}$ Erlenmeyer flask and grown at $28^{\circ} \mathrm{C}$ for 15 hours.

The E. coli and yeast cells prepared as described above were harvested by centrifugation at $3500 \mathrm{rpm}$ for $5 \mathrm{~min}$. Then, the $E$. coli and $S$. cerevisiae cells were mixed and resuspended in $5 \mathrm{ml}$ of $\mathrm{XY}$ media $\left(\mathrm{OD}_{600} \sim 8.0\right)$ and cultured in $100-\mathrm{ml}$ shake flask at $22^{\circ} \mathrm{C}$ and $220 \mathrm{rpm}$ for 60 or 72 hours (final $\mathrm{OD}_{600} \sim 40$ ). In the case of CL production, the parent $S$. cerevisiae strain (CEN.PK2-1D) was precultivated in YPD and mixed with the CL-producing E. coli cells.

\section{Isolation and characterization of SLs and their precursors}

Unless specified, $5 \mathrm{ml}$ of culture was used for compound extraction. For the extraction of intracellular and extracellular metabolites, $5 \mathrm{ml}$ of cell culture was transferred into $50-\mathrm{ml}$ centrifuge tube, and the cells and medium were separated by centrifugation at $5000 \mathrm{rpm}$ for $10 \mathrm{~min}$.

The cell pellets were transferred to a new $2-\mathrm{ml}$ microcentrifuge tube and resuspended in $150 \mu$ l of dimethylformamide and shaken vigorously, followed by the addition of $850 \mu$ l of acetone and vigorous shaking for $15 \mathrm{~min}$ by using a vortex mixer (Thermo Fisher Scientific) and centrifugation at 12,000 rpm for $10 \mathrm{~min}$. Then, the supernatant was transferred to a new $1.7-\mathrm{ml}$ microcentrifuge tube and dried in a vacuum concentrator (Eppendorf Vacufuge plus) at $30^{\circ} \mathrm{C}$ for 2 to 3 hours. The dried sample was dissolved in $100 \mu \mathrm{l}$ of acetone.
The medium was transferred into a 50-ml centrifuge tube containing $4 \mathrm{ml}$ of ethyl acetate. The mixture was vortexed vigorously for 5 min by using a vortex mixer (Genie) and then centrifugated at $4000 \mathrm{rpm}$ for $20 \mathrm{~min}$. The upper ethyl acetate layer of extracted medium was transferred into a $1.7-\mathrm{ml}$ microcentrifuge tube and evaporated to dryness by using a vacuum concentrator (Eppendorf Vacufuge plus) at $30^{\circ} \mathrm{C}$ for $30 \mathrm{~min}$. The dried extract was dissolved in $100 \mu$ l of acetone.

The samples were centrifuged at 12,000 rpm for $10 \mathrm{~min}$ before applying to high-performance LC (HPLC) analysis. Both UV-VIS and MS detectors were used. The used instrument was Shimadzu LC-MS 2020 (Kyoto, Japan) or a Waters UPLC (ACQUITY) coupled with a Bruker quadrupole time-of-flight MS (Q-TOF MS; micrOTOF II).

AT $\beta C$ and $9 \mathrm{C} \beta C$ were analyzed based on Separation Method I and a $\mathrm{C}_{18}$ column (Kinetex C18, $100 \mathrm{~mm}$ by $2.1 \mathrm{~mm}, 100 \AA$, particle size of $2.6 \mu \mathrm{m}$; Phenomex, Torrance, CA, USA). In Separation Method I, the column temperature was $25^{\circ} \mathrm{C}$, single mobile phase was $0.1 \%(\mathrm{v} / \mathrm{v})$ formic acid in methanol, the isocratic elution was $0.4 \mathrm{ml} / \mathrm{min}$, and the analysis time was $20 \mathrm{~min}$. The injection volume was $10 \mu \mathrm{l}$, and the UV-VIS absorption was monitored in the range of 190 to $800 \mathrm{~nm}$. With Separation Method I, the retention time of $9 \mathrm{C} \beta \mathrm{C}$ was $7.78 \mathrm{~min}$ and that of $\mathrm{AT} \beta \mathrm{C}$ was $8.17 \mathrm{~min}$ (characteristic absorption wavelengths: 447 and $471 \mathrm{~nm}$ for both carotenes).

9C $\beta A 10^{\prime} \mathrm{COL}$ was analyzed using Separation Method II and a $\mathrm{C}_{18}$ column (Poroshell $120 \mathrm{EC}-\mathrm{C} 18,100 \mathrm{~mm}$ by $3.0 \mathrm{~mm}, 100 \AA$, particle size $2.7 \mu \mathrm{m}$; Aglient, Santa Clara, CA, USA). In Separation Method II, the column temperature was $40^{\circ} \mathrm{C}$, the mobile phase A was $0.1 \%(\mathrm{v} / \mathrm{v})$ formic acid in water, the mobile phase B was $0.1 \%$ $(\mathrm{v} / \mathrm{v})$ formic acid in methanol, and the gradient elution was $0.5 \mathrm{ml} / \mathrm{min}$. The gradient was as follows: 0 to $18 \mathrm{~min}, 5$ to $100 \% \mathrm{~B}$; 18 to $43 \mathrm{~min}$, $100 \% \mathrm{~B}$; and 43 to $45 \mathrm{~min}, 100$ to $5 \% \mathrm{~B}$. The injection volume was $10 \mu \mathrm{l}$, and the UV-VIS absorption was monitored in the range of 190 to $800 \mathrm{~nm}$. With Separation Method II, the retention time of 9C $\beta A 10^{\prime} \mathrm{COL}$ was $12.64 \mathrm{~min}$ (characteristic absorption wavelengths: 373 and $390 \mathrm{~nm})$.

All the SLs (CL, CLA, putative 18-hydroxy-CLA, 4DO, 5DS, and $\mathrm{OB})$ were analyzed using Separation Method III and a $\mathrm{C}_{18}$ column (Kinetex C18, $100 \mathrm{~mm}$ by $2.1 \mathrm{~mm}, 100 \AA$, particle size $2.6 \mu \mathrm{m}$; Phenomex, Torrance, CA, USA). In Separation Method III, the column temperature was $40^{\circ} \mathrm{C}$, the mobile phase $A$ was $0.1 \%(\mathrm{v} / \mathrm{v})$ formic acid in water, the mobile phase B was $0.1 \%(\mathrm{v} / \mathrm{v})$ formic acid in acetonitrile, and the gradient elution was $0.4 \mathrm{ml} / \mathrm{min}$. The gradient was as follows: 0 to $28 \mathrm{~min}, 5$ to $100 \% \mathrm{~B} ; 28$ to $35 \mathrm{~min}, 100 \% \mathrm{~B}$; and 35 to $40 \mathrm{~min}, 5 \% \mathrm{~B}$. The injection volume was $10 \mu \mathrm{l}$, and the UV-VIS absorption was monitored in the range of 190 to $800 \mathrm{~nm}$. With Separation Method III, the retention time and the characteristic absorption wavelength of various analytes are listed as follows: $\beta \mathrm{I}$, $15.91 \mathrm{~min}(298 \mathrm{~nm})$; CL, $20.58 \mathrm{~min}(269 \mathrm{~nm})$; CLA, $16.32 \mathrm{~min}(271 \mathrm{~nm})$; 18-hydroxy-CLA, $12.16 \mathrm{~min}$; 4DO, $15.81 \mathrm{~min}$; OB, $11.03 \mathrm{~min}$; and 5DS, $15.97 \mathrm{~min}$. The compounds without the wavelength information were detected using an MS detector, which operates in the $\mathrm{m} / z$ range of 50 to 800 in the positive or negative ion modes. Electrospray ionization (ESI) was used. The desolvation line temperature was $250^{\circ} \mathrm{C}$. The nebulizing gas and drying gas flow rates were 1.5 and 15 liter/min, respectively.

The HR-MS analysis of CLA was performed by using an Ultra Performance LC (UPLC, Waters ACQUITY) linked with a TOF MS (Bruker micrOTOF II). The separation was based on Separation 
Method IV and a C18 column (Poroshell 120 EC-C18 column, $2.1 \mathrm{~mm}$ by $50 \mathrm{~mm}$, particle size of $2.7 \mu \mathrm{m}$; Agilent Technologies). In Separation Method IV, the column temperature was $40^{\circ} \mathrm{C}$, the mobile phase A was $0.1 \%(\mathrm{v} / \mathrm{v})$ formic acid in water, the mobile phase $\mathrm{B}$ was $0.1 \%(\mathrm{v} / \mathrm{v})$ formic acid in acetonitrile, and gradient elution was $0.3 \mathrm{ml} / \mathrm{min}$. The gradient was as follows: 0 to $15 \mathrm{~min}, 20$ to $100 \% \mathrm{~B} ; 15$ to $20 \mathrm{~min}, 100$ to $20 \% \mathrm{~B}$; and 20 to $22 \mathrm{~min}, 20 \% \mathrm{~B}$. The MS/MS analysis was performed in product ion scan mode based on ESI (negative mode). The optimized MS/MS conditions were as follows: capillary voltage, $3500 \mathrm{~V}$; end plate offset, $500 \mathrm{~V}$; desolvation gas flow rate $\left(\mathrm{N}_{2}\right), 4.0$ liter/min; drying gas temperature, $200^{\circ} \mathrm{C}$; hexapole RF, $50 \mathrm{Vpp}$; ion energy, $4 \mathrm{eV}$; collision energy, $18 \mathrm{eV}$; and precursor ion $\mathrm{m} / \mathrm{z}, 331.2$. The scan range of $\mathrm{m} / \mathrm{z}$ was from 50 to 1300 . Sodium formate $(10 \mathrm{mM})$ solution was used to calibrate the MS before every use. HR-MS analysis of 18-hydroxy-CLA was performed on a Synapt G2-Si Q-TOF MS (Waters) coupled to an I-class UPLC system (Waters). The separation was conducted using Separation Method III and the $\mathrm{C}_{18}$ column (Kinetex C18, $100 \mathrm{~mm}$ by $2.1 \mathrm{~mm}$, $100 \AA$, particle size $2.6 \mu \mathrm{m}$; Phenomex, Torrance, CA, USA) as mentioned above. The injection volume was $5 \mu$ l. The MS were obtained using the negative ion mode, the scan range of $\mathrm{m} / \mathrm{z}$ was from 50 to 1200 with a $0.2 \mathrm{~s}$ scan time. MS/MS was acquired in a continuum data format and data-dependent fashion with collision energy $25 \mathrm{eV}$. Source and desolvation temperatures were $150^{\circ}$ and $600^{\circ} \mathrm{C}$, respectively. Desolvation gas was set to 1100 liter/hour, and cone gas was set to 150 liter/hour. All gases were nitrogen except the collision gas, which was argon. Capillary voltage was $1.5 \mathrm{kV}$ in negative ion mode.

In the experiment aiming to improve 5DS biosynthesis, $\beta \mathrm{I}$ was analyzed without drying to avoid evaporation loss. A total of $0.5 \mathrm{ml}$ of cell culture was transferred into a 2-ml Eppendorf Safe-Lock Tubes containing $0.1 \mathrm{~g}$ of glass beads (Sigma-Aldrich, G8772) and $0.5 \mathrm{ml}$ of ethyl acetate. The mixture was incubated at $25^{\circ} \mathrm{C}$ and $1500 \mathrm{rpm}$ for 1 hour by using a ThermoMixer (Eppendorf) and then centrifuged at 14,000 rpm for $10 \mathrm{~min}$. One hundred microliters of the upper organic phase was used for gas chromatography-MS (5977B GC/MSD, Agilent Technologies) analysis. Five microliters of the filtered sample was injected in a spitless mode. HP-5MS capillary column ( $30 \mathrm{~m}$ by $0.25 \mathrm{~mm}, 0.25-\mu \mathrm{m}$ film thickness; Agilent Technologies) was used, with helium as the carrier gas at the flow rate of $1 \mathrm{ml} / \mathrm{min}$. The following oven temperature program was carried out: $50^{\circ} \mathrm{C}$ for $1 \mathrm{~min}, 50^{\circ}$ to $100^{\circ} \mathrm{C}$ at a rate of $5^{\circ} \mathrm{C} / \mathrm{min}, 100^{\circ}$ to $300^{\circ} \mathrm{C}$ at a rate of $50^{\circ} \mathrm{C} / \mathrm{min}$, and $300^{\circ} \mathrm{C}$ for $1 \mathrm{~min}$. Commercially available $\beta$ I (Sigma-Aldrich, I12603) was used to prepare standard solutions. The retention time was $11.02 \mathrm{~min}$.

In the experiment aiming to improve 5DS biosynthesis, 5DS was quantified by using the same procedure as the HR-MS experiment, except the used mode was scan instead of product ion scan. No collision energy was applied. The quantification was based on extracted ion chromatogram $(\mathrm{m} / \mathrm{z}: 331.20 \pm 0.01)$. The retention time was $7.95 \mathrm{~min}$.

In the experiment aiming to improve 5DS biosynthesis, the quantification of $A T \beta C$ and $9 C \beta C$ was done by using Separation Method V and a C30 column (YMC Carotenoid, $250 \mathrm{~mm}$ by $4.6 \mathrm{~mm}$, $5 \mu \mathrm{m})$. In Separation Method V, the column temperature was $30^{\circ} \mathrm{C}$; the single mobile phase was $75 \%(\mathrm{v} / \mathrm{v})$ ethanol, $20 \%(\mathrm{v} / \mathrm{v})$ methanol, and $5 \%(\mathrm{v} / \mathrm{v})$ tetrahydrofuran; and the isocratic elution was $1 \mathrm{ml} / \mathrm{min}$ for $20 \mathrm{~min}$. The injection volume was $10 \mu \mathrm{l}$, and the used detector was a UV-VIS detector $(475 \mathrm{~nm})$. The model of the HPLC was Agilent 1260 Infinity. The retention time of $A T \beta C$ and $9 \mathrm{C} \beta C$ was 12.26 and $13.98 \mathrm{~min}$, respectively.
In the experiment aiming to improve 5DS biosynthesis, the quantification of CL was done using Separation Method III on Agilent 1260 Infinity HPLC with a UV detector. The column was Poroshell 120 EC-C18 (150 mm by $4.6 \mathrm{~mm}, 100 \AA$ A, particle size of $4 \mu \mathrm{m}$; Agilent Technologies). The injection volume was $10 \mu \mathrm{l}$. The UV detector with a wavelength of $269 \mathrm{~nm}$ was used. The retention time of CL was $31.72 \mathrm{~min}$.

\section{SUPPLEMENTARY MATERIALS}

Supplementary material for this article is available at https://science.org/doi/10.1126/ sciadv.abh4048

View/request a protocol for this paper from Bio-protocol.

\section{REFERENCES AND NOTES}

1. C. E. Cook, L. P. Whichard, B. Turner, M. E. Wall, G. H. Egley, Germination of witchweed (Striga lutea Lour.): Isolation and properties of a potent stimulant. Science 154, 1189-1190 (1966).

2. K. Akiyama, K. Matsuzaki, H. Hayashi, Plant sesquiterpenes induce hyphal branching in arbuscular mycorrhizal fungi. Nature 435, 824-827 (2005).

3. S. Al-Babili, H. J. Bouwmeester, Strigolactones, a novel carotenoid-derived plant hormone. Annu. Rev. Plant Biol. 66, 161-186 (2015).

4. M. Vurro, C. Prandi, F. Baroccio, Strigolactones: How far is their commercial use for agricultural purposes? Pest Manag. Sci. 72, 2026-2034 (2016).

5. S. M. Smith, Q\&A: What are strigolactones and why are they important to plants and soil microbes? BMC Biol. 12, 19 (2014).

6. R. J. Chesterfield, C. E. Vickers, C. A. Beveridge, Translation of strigolactones from plant hormone to agriculture: Achievements, future perspectives, and challenges. Trends Plant Sci. 25, 1087-1106 (2020).

7. E. B. Aliche, C. Screpanti, A. De Mesmaeker, T. Munnik, H. J. Bouwmeester, Science and application of strigolactones. New Phytol. 227, 1001-1011 (2020).

8. K. Yoneyama, Recent progress in the chemistry and biochemistry of strigolactones. J. Pestic. Sci. 45, 45-53 (2020).

9. B. Zwanenburg, T. Pospiśsil, Structure and activity of strigolactones: New plant hormones with a rich future. Mol. Plant 6, 38-62 (2013).

10. M. T. Waters, C. Gutjahr, T. Bennett, D. C. Nelson, Strigolactone signaling and evolution. Annu. Rev. Plant Biol. 68, 291-322 (2017).

11. S. Abe, A. Sado, K. Tanaka, T. Kisugi, K. Asami, S. Ota, H. I. Kim, K. Yoneyama, X. Xie, T. Ohnishi, Y. Seto, S. Yamaguchi, K. Akiyama, K. Yoneyama, T. Nomura, Carlactone is converted to carlactonoic acid by MAX1 in Arabidopsis and its methyl ester can directly interact with AtD14 in vitro. Proc. Natl. Acad. Sci. U.S.A. 111, 18084-18089 (2014).

12. K. Ueno, T. Furumoto, S. Umeda, M. Mizutani, H. Takikawa, R. Batchvarova, Y. Sugimoto, Heliolactone, a non-sesquiterpene lactone germination stimulant for root parasitic weeds from sunflower. Phytochemistry 108, 122-128 (2014).

13. H. I. Kim, T. Kisugi, P. Khetkam, X. Xie, K. Yoneyama, K. Uchida, T. Yokota, T. Nomura, C. S. P. McErlean, K. Yoneyama, Avenaol, a germination stimulant for root parasitic plants from Avena strigosa. Phytochemistry 103, 85-88 (2014).

14. T. V. Charnikhova, K. Gaus, A. Lumbroso, M. Sanders, J. P. Vincken, A. De Mesmaeker, C. P. Ruyter-Spira, C. Screpanti, H. J. Bouwmeester, Zealactones. Novel natural strigolactones from maize. Phytochemistry 137, 123-131 (2017).

15. X. Xie, N. Mori, K. Yoneyama, T. Nomura, K. Uchida, K. Yoneyama, K. Akiyama, Lotuslactone, a non-canonical strigolactone from Lotus japonicus. Phytochemistry 157, 200-205 (2019).

16. A. Alder, M. Jamil, M. Marzorati, M. Bruno, M. Vermathen, P. Bigler, S. Ghisla, H. Bouwmeester, P. Beyer, S. Al-Babili, The path from $\beta$-carotene to carlactone, a strigolactone-like plant hormone. Science 335, 1348-1351 (2012).

17. H. Lin, R. Wang, Q. Qian, M. Yan, X. Meng, Z. Fu, C. Yan, B. Jiang, Z. Su, J. Li, Y. Wang, DWARF27, an iron-containing protein required for the biosynthesis of strigolactones, regulates rice tiller bud outgrowth. Plant Cell 21, 1512-1525 (2009).

18. J. T. Vogel, M. H. Walter, P. Giavalisco, A. Lytovchenko, W. Kohlen, T. Charnikhova, A. J. Simkin, C. Goulet, D. Strack, H. J. Bouwmeester, A. R. Fernie, H. J. Klee, SICCD7 controls strigolactone biosynthesis, shoot branching and mycorrhiza-induced apocarotenoid formation in tomato. Plant J. 61, 300-311 (2010).

19. S. H. Schwartz, X. Qin, M. C. Loewen, The biochemical characterization of two carotenoid cleavage enzymes from Arabidopsis indicates that a carotenoid-derived compound inhibits lateral branching. J. Biol. Chem. 279, 46940-46945 (2004).

20. M. Bruno, M. Vermathen, A. Alder, F. Wüst, P. Schaub, R. van der Steen, P. Beyer, S. Ghisla, S. Al-Babili, Insights into the formation of carlactone from in-depth analysis of the CCD8catalyzed reactions. FEBS Lett. 591, 792-800 (2017).

21. M. Lopez-Obando, Y. Ligerot, S. Bonhomme, F. D. Boyer, C. Rameau, Strigolactone biosynthesis and signaling in plant development. Development 142, 3615-3619 (2015). 
22. K. Yoneyama, N. Mori, T. Sato, A. Yoda, X. Xie, M. Okamoto, M. Iwanaga, T. Ohnishi, H. Nishiwaki, T. Asami, T. Yokota, K. Akiyama, K. Yoneyama, T. Nomura, Conversion of carlactone to carlactonoic acid is a conserved function of MAX1 homologs in strigolactone biosynthesis. New Phytol. 218, 1522-1533 (2018).

23. X. Xie, K. Yoneyama, T. Kisugi, K. Uchida, S. Ito, K. Akiyama, H. Hayashi, T. Yokota, T. Nomura, K. Yoneyama, Confirming stereochemical structures of strigolactones produced by rice and tobacco. Mol. Plant 6, 153-163 (2013).

24. Y. Zhang, A. D. J. van Dijk, A. Scaffidi, G. R. Flematti, M. Hofmann, T. Charnikhova, F. Verstappen, J. Hepworth, S. van der Krol, O. Leyser, S. M. Smith, B. Zwanenburg S. Al-Babili, C. Ruyter-Spira, H. J. Bouwmeester, Rice cytochrome P450 MAX1 homologs catalyze distinct steps in strigolactone biosynthesis. Nat. Chem. Biol. 10, 1028-1033 (2014).

25. K. Ueno, H. Nakashima, M. Mizutani, H. Takikawa, Y. Sugimoto, Bioconversion of 5-deoxystrigol stereoisomers to monohydroxylated strigolactones by plants. J. Pestic. Sci. 43, 198-206 (2018).

26. M. Iseki, K. Shida, K. Kuwabara, T. Wakabayashi, M. Mizutani, H. Takikawa, Y. Sugimoto, Evidence for species-dependent biosynthetic pathways for converting carlactone to strigolactones in plants. J. Exp. Bot. 69, 2305-2318 (2018).

27. T. Wakabayashi, M. Hamana, A. Mori, R. Akiyama, K. Ueno, K. Osakabe, Y. Osakabe, H. Suzuki, H. Takikawa, M. Mizutani, Y. Sugimoto, Direct conversion of carlactonoic acid to orobanchol by cytochrome P450 CYP722C in strigolactone biosynthesis. Sci. Adv. 5 eaax9067 (2019).

28. T. Wakabayashi, K. Shida, Y. Kitano, H. Takikawa, M. Mizutani, Y. Sugimoto, CYP722C from Gossypium arboreum catalyzes the conversion of carlactonoic acid to 5-deoxystrigol. Planta 251, 97 (2020).

29. C. H. Walker, K. Siu-Ting, A. Taylor, M. J. O'Connell, T. Bennett, Strigolactone synthesis is ancestral in land plants, but canonical strigolactone signalling is a flowering plant innovation. BMC Biol. 17, 70 (2019).

30. P. M. Delaux, X. Xie, R. E. Timme, V. Puech-Pages, C. Dunand, E. Lecompte, C. F. Delwiche, K. Yoneyama, G. Bécard, N. Séjalon-Delmas, Origin of strigolactones in the green lineage. New Phytol. 195, 857-871 (2012).

31. P. J. Harrison, S. A. Newgas, F. Descombes, S. A. Shepherd, A. J. Thompson, T. D. Bugg Biochemical characterization and selective inhibition of $\beta$-carotene cis-trans isomerase D27 and carotenoid cleavage dioxygenase CCD8 on the strigolactone biosynthetic pathway. FEBS J. 282, 3986-4000 (2015).

32. R. Verwaal, J. Wang, J. P. Meijnen, H. Visser, G. Sandmann, J. A. van den Berg, A. J. J. van Ooyen, High-level production of beta-carotene in Saccharomyces cerevisiae by successive transformation with carotenogenic genes from Xanthophyllomyces dendrorhous. Appl. Environ. Microbiol. 73, 4342-4350 (2007).

33. N. Werner, C. A. Ramirez-Sarmiento, E. Agosin, Protein engineering of carotenoid cleavage dioxygenases to optimize $\beta$-ionone biosynthesis in yeast cell factories. Food Chem. 299, 125089 (2019).

34. M. K. Dhar, S. Mishra, A. Bhat, S. Chib, S. Kaul, Plant carotenoid cleavage oxygenases: Structure-function relationships and role in development and metabolism. Brief. Funct. Genom. 19, 1-9 (2020).

35. F. X. Cunningham Jr., E. Gantt, A study in scarlet: Enzymes of ketocarotenoid biosynthesis in the flowers of Adonis aestivalis. Plant J. 41, 478-492 (2005)

36. F. C. Huang, P. Molnár, W. Schwab, Cloning and functional characterization of carotenoid cleavage dioxygenase 4 genes. J. Exp. Bot. 60, 3011-3022 (2009)

37. K. Yoneyama, X. Xie, K. Yoneyama, T. Kisugi, T. Nomura, Y. Nakatani, K. Akiyama, C. S.P. McErlean, Which are the major players, canonical or non-canonical strigolactones? J. Exp. Bot. 69, 2231-2239 (2018)

38. R. Halouzka, P. Tarkowski, B. Zwanenburg, S. Ćavar Zeljković, Stability of strigolactone analog GR24 toward nucleophiles. Pest Manag. Sci. 74, 896-904 (2018).

39. A. Zhou, K. Zhou, Y. Li, Rational design strategies for functional reconstitution of plant cytochrome P450s in microbial systems. Curr. Opin. Plant Biol. 60, 102005 (2021).

40. B. W. Biggs, C. G. Lim, K. Sagliani, S. Shankar, G. Stephanopoulos, M. De Mey, P. K. Ajikumar, Overcoming heterologous protein interdependency to optimize P450-mediated Taxol precursor synthesis in Escherichia coli. Proc. Natl. Acad. Sci. U.S.A. 113, 3209-3214 (2016).

41. K. Ueno, S. Nomura, S. Muranaka, M. Mizutani, H. Takikawa, Y. Sugimoto, Ent-2'-epiOrobanchol and its acetate, as germination stimulants for Striga gesnerioides seeds isolated from cowpea and red clover. J. Agric. Food Chem. 59, 10485-10490 (2011).

42. J. A. López-Ráez, T. Charnikhova, V. Gómez-Roldán, R. Matusova, W. Kohlen, R. De Vos, F. Verstappen, V. Puech-Pages, G. Bécard, P. Mulder, H. J. Bouwmeester, Tomato strigolactones are derived from carotenoids and their biosynthesis is promoted by phosphate starvation. New Phytol. 178, 863-874 (2008).

43. N. Mori, T. Nomura, K. Akiyama, Identification of two oxygenase genes involved in the respective biosynthetic pathways of canonical and non-canonical strigolactones in Lotus japonicus. Planta 251, 40 (2020).

44. P. K. Ajikumar, W.-H. Xiao, K. E. Tyo, Y. Wang, F. Simeon, E. Leonard, O. Mucha, T. H. Phon, B. Pfeifer, G. Stephanopoulos, Isoprenoid pathway optimization for Taxol precursor overproduction in Escherichia coli. Science 330, 70-74 (2010).
45. K. Zhou, R. Zou, G. Stephanopoulos, H.-P. Too, Metabolite profiling identified methylerythritol cyclodiphosphate efflux as a limiting step in microbial isoprenoid production. PLOS ONE 7, e47513 (2012).

46. D. C. Volke, J. Rohwer, R. Fischer, S. Jennewein, Investigation of the methylerythritol 4-phosphate pathway for microbial terpenoid production through metabolic control analysis. Microb. Cell Fact. 18, 192 (2019).

47. G. Daletos, C. Katsimpouras, G. Stephanopoulos, Novel strategies and platforms for industrial isoprenoid engineering. Trends Biotechnol. 38, 811-822 (2020).

48. H. Liang, X. Ma, W. Ning, Y. Liu, A. J. Sinskey, G. Stephanopoulos, K. Zhou, X. Ma, W. Ning Y. Liu, A. J. Sinskey, G. Stephanopoulos, K. Zhou, Constructing an ethanol utilization pathway in Escherichia coli to produce acetyl-CoA derived compounds. Metab. Eng. 65 223-231 (2021).

49. Y. Wang, H. J. Bouwmeester, Structural diversity in the strigolactones. J. Exp. Bot. 69 2219-2230 (2018).

50. K. Mashiguchi, Y. Seto, S. Yamaguchi, Strigolactone biosynthesis, transport and perception. Plant J. 105, 335-350 (2021)

51. D. Sato, A. A. Awad, S. H. Chae, T. Yokota, Y. Sugimoto, Y. Takeuchi, K. Yoneyama, Analysis of strigolactones, germination stimulants for Striga and Orobanche, by high-performance liquid chromatography/tandem mass spectrometry. J. Agric. Food Chem. 51, 1162-1168 (2003).

52. A. J. Humphrey, M. H. Beale, Strigol: Biogenesis and physiological activity. Phytochemistry 67, 636-640 (2006)

53. B. Zwanenburg, S. Ćavar Zeljković, T. Pospišil, Synthesis of strigolactones, a strategic account. Pest Manag. Sci. 72, 15-29 (2016).

54. C. Ruyter-Spira, W. Kohlen, T. Charnikhova, A. van Zeijl, L. van Bezouwen, N. de Ruijter, C. Cardoso, J. A. Lopez-Raez, R. Matusova, R. Bours, F. Verstappen, H. Bouwmeester, Physiological effects of the synthetic strigolactone analog GR24 on root system architecture in Arabidopsis: Another belowground role for strigolactones? Plant Physiol. 155, 721-734 (2011).

55. A. Scaffidi, M. T. Waters, Y. K. Sun, B. W. Skelton, K. W. Dixon, E. L. Ghisalberti, G. R. Flematti, S. M. Smith, Strigolactone hormones and their stereoisomers signal through two related receptor proteins to induce different physiological responses in Arabidopsis. Plant Physiol. 165, 1221-1232 (2014).

56. K. Yoneyama, A. A. Awad, X. Xie, K. Yoneyama, Y. Takeuchi, Strigolactones as germination stimulants for root parasitic plants. Plant Cell Physiol. 51, 1095-1103 (2010).

57. K. Akiyama, S. Ogasawara, S. Ito, H. Hayashi, Structural requirements of strigolactones for hyphal branching in AM fungi. Plant Cell Physiol. 51, 1104-1117 (2010).

58. B. Zwanenburg, D. Blanco-Ania, Strigolactones: New plant hormones in the spotlight J. Exp. Bot. 69, 2205-2218 (2018)

59. F. D. Boyer, A. de Saint Germain, J. P. Pillot, J. B. Pouvreau, V. X. Chen, S. Ramos, A. Stévenin, P. Simier, P. Delavault, J. M. Beau, C. Rameau, Structure-activity relationship studies of strigolactone-related molecules for branching inhibition in garden pea: Molecule design for shoot branching. Plant Physiol. 159, 1524-1544 (2012).

60. K. Yoneyama, R. Arakawa, K. Ishimoto, H. I. Kim, T. Kisugi, X. Xie, T. Nomura, F. Kanampiu, T. Yokota, T. Ezawa, K. Yoneyama, Difference in Striga-susceptibility is reflected in strigolactone secretion profile, but not in compatibility and host preference in arbuscular mycorrhizal symbiosis in two maize cultivars. New Phytol. 206, 983-989 (2015).

61. T. Wakabayashi, S. Ishiwa, K. Shida, N. Motonami, H. Suzuki, H. Takikawa, M. Mizutani, Y. Sugimoto, Identification and characterization of sorgomol synthase in sorghum strigolactone biosynthesis. Plant Physiol. 185, 902-913 (2021).

62. P. B. Brewer, K. Yoneyama, F. Filardo, E. Meyers, A. Scaffidi, T. Frickey, K. Akiyama, Y. Seto, E. A. Dun, J. E. Cremer, S. C. Kerr, M. T. Waters, G. R. Flematti, M. G. Mason, G. Weiller, S. Yamaguchi, T. Nomura, S. M. Smith, K. Yoneyama, C. A. Beveridge, LATERAL BRANCHING OXIDOREDUCTASE acts in the final stages of strigolactone biosynthesis in Arabidopsis. Proc. Natl. Acad. Sci. U.S.A. 113, 6301-6306 (2016).

63. K. Yoneyama, K. Akiyama, P. B. Brewer, N. Mori, M. Kawano-Kawada, S. Haruta, H. Nishiwaki, S. Yamauchi, X. Xie, M. Umehara, C. A. Beveridge, K. Yoneyama, T. Nomura, Hydroxyl carlactone derivatives are predominant strigolactones in Arabidopsis. Plant Direct 4, e00219 (2020).

64. H. Proust, B. Hoffmann, X. Xie, K. Yoneyama, D. G. Schaefer, K. Yoneyama, F. Nogué, C. Rameau, Strigolactones regulate protonema branching and act as a quorum sensing-like signal in the moss Physcomitrella patens. Development 138, 1531-1539 (2011).

65. E. L. Decker, A. Alder, S. Hunn, J. Ferguson, M. T. Lehtonen, B. Scheler, K. L. Kerres, G. Wiedemann, V. Safavi-Rizi, S. Nordzieke, A. Balakrishna, L. Baz, J. Avalos, J. P. T. Valkonen, R. Reski, S. Al-Babili, Strigolactone biosynthesis is evolutionarily conserved, regulated by phosphate starvation and contributes to resistance against phytopathogenic fungi in a moss, Physcomitrella patens. New Phytol. 216, 455-468 (2017)

66. A. de Saint Germain, S. Bonhomme, F. D. Boyer, C. Rameau, Novel insights into strigolactone distribution and signalling. Curr. Opin. Plant Biol. 16, 583-589 (2013). 
67. J. Zhang, X.-X. Fu, R.-Q. Li, X. Zhao, Y. Liu, M.-H. Li, A. Zwaenepoel, H. Ma, B. Goffinet, Y.-L. Guan, J.-Y. Xue, Y.-Y. Liao, Q.-F. Wang, Q.-H. Wang, J.-Y. Wang, G.-Q. Zhang, Z.-W. Wang, Y. Jia, M.-Z. Wang, S.-S. Dong, J.-F. Yang, Y.-N. Jiao, Y.-L. Guo, H.-Z. Kong, A.-M. Lu, H.-M. Yang, S.-Z. Zhang, Y. Van de Peer, Z.-J. Liu, Z.-D. Chen, The hornwort genome and early land plant evolution. Nat. Plants 6, 107-118 (2020).

68. A. O. Chatzivasileiou, V. Ward, S. M. Edgar, G. Stephanopoulos, Two-step pathway for isoprenoid synthesis. Proc. Natl. Acad. Sci. U.S.A. 116, 506-511 (2019).

69. S. Alberti, A. D. Gitler, S. Lindquist, A suite of Gateway cloning vectors for highthroughput genetic analysis in Saccharomyces cerevisiae. Yeast 24, 913-919 (2007).

70. D. G. Gibson, L. Young, R.-Y. Chuang, J. C. Venter, C. A. Hutchison III, H. O. Smith, Enzymatic assembly of DNA molecules up to several hundred kilobases. Nat. Methods 6 343-345 (2009).

71. Z. Shao, H. Zhao, H. Zhao, DNA assembler, an in vivo genetic method for rapid construction of biochemical pathways. Nucleic Acids Res. 37, e16 (2009).

72. M. E. Auldridge, A. Block, J. T. Vogel, C. Dabney-Smith, I. Mila, M. Bouzayen, M. Magallanes-Lundback, D. DellaPenna, D. R. McCarty, H. J. Klee, Characterization of three members of the Arabidopsis carotenoid cleavage dioxygenase family demonstrates the divergent roles of this multifunctional enzyme family. Plant J. 45, 982-993 (2006).

73. V. F. Cataldo, N. Arenas, V. Salgado, C. Camilo, F. Ibáñez, E. Agosin, Heterologous production of the epoxycarotenoid violaxanthin in Saccharomyces cerevisiae. Metab. Eng. 59, 53-63 (2020).

74. J. J. Braymer, R. Lill, Iron-sulfur cluster biogenesis and trafficking in mitochondria. J. Biol. Chem. 292, 12754-12763 (2017).

75. N. Peeters, I. Small, Dual targeting to mitochondria and chloroplasts. Biochim. Biophys. Acta 1541, 54-63 (2001).

76. J. Yuan, C. B. Ching, Mitochondrial acetyl-CoA utilization pathway for terpenoid productions. Metab. Eng. 38, 303-309 (2016).

77. Y. Zhang, E. R. Lyver, E. Nakamaru-Ogiso, H. Yoon, B. Amutha, D. W. Lee, E. Bi, T. Ohnishi, F. Daldal, D. Pain, A. Dancis, Dre2, a conserved eukaryotic Fe/S cluster protein, functions in cytosolic Fe/S protein biogenesis. Mol. Cell. Biol. 28, 5569-5582 (2008).

78. M. Manicki, J. Majewska, S. Ciesielski, B. Schilke, A. Blenska, J. Kominek, J. Marszalek, E. A. Craig, R. Dutkiewicz, Overlapping binding sites of the frataxin homologue assembly factor and the heat shock protein 70 transfer factor on the Isu iron-sulfur cluster scaffold protein. J. Biol. Chem. 289, 30268-30278 (2014).
79. Y. Li, C. D. Smolke, Engineering biosynthesis of the anticancer alkaloid noscapine in yeast. Nat. Commun. 7, 12137 (2016)

80. S. Xu, C. Chen, Y. Li, Engineering of phytosterol-producing yeast platforms for functional reconstitution of downstream biosynthetic pathways. ACS Synth. Biol. 9, 3157-3170 (2020).

81. K.-D. Entian, P. Kötter, 25 Yeast genetic strain and plasmid collections. Methods Microbiol. 36, 629-666 (2007)

Acknowledgments: pAC-BETAipi was a gift from F. X. Cunningham Jr. (Addgene plasmid no. 53277; http://n2t.net/addgene:53277; RRID: Addgene_53277). We thank the Metabolomics Core Facility at UC Riverside and A. Bhatia for instrument access, training, and data analysis. We thank T. Chiu for valuable feedback in the preparation of the manuscript. Funding: This work is supported by the Cancer Research Coordinating Committee Research Award (grant to Y.L., CRN-20-634571), the NIH Director's New Innovator Award (grant to Y.L., DP2-AT011445), the Plants3D National Science Foundation Research Traineeship Program (grant to A.Z. and A.V., 1922642), the Ministry of Education Singapore (Grant identifier: R-279-000-594-112, grants to K.Z.), and the National Research Foundation Singapore [project IDs: R-279-000-587592 (through DiSTAP program) and R-279-000-512-281, grants to K.Z.]. Author contributions: S.W., K.Z., and Y.L. conceived the project. S.W., X.M., A.Z., K.Z., and Y.L. designed the experiments. S.W., X.M., A.Z., and A.V. performed the experiments and analyzed the results. S.W., X.M., A.Z., K.Z., and Y.L. wrote the manuscript. Competing interests: Y.L. and S.W. are inventors on a provisional patent application related to this work filed by University of California, Riverside (U.S. Provisional Application No. 63/142,801, filed 28 January 2021, patent pending). The authors declare that they have no other competing interests. Data and materials availability: All data needed to evaluate the conclusions in the paper are present in the paper and/or the Supplementary Materials.

Submitted 8 March 2021

Accepted 27 July 2021

Published 17 September 2021

$10.1126 /$ sciadv.abh4048

Citation: S. Wu, X. Ma, A. Zhou, A. Valenzuela, K. Zhou, Y. Li, Establishment of strigolactone-producing bacterium-yeast consortium. Sci. Adv. 7, eabh4048 (2021). 